\title{
HUBBLE SPACE TELESCOPE SPECTROSCOPY OF BROWN DWARFS DISCOVERED WITH THE WIDE-FIELD INFRARED SURVEY EXPLORER
}

\author{
Adam C. Schneider ${ }^{1}$, Michael C. Cushing ${ }^{1}$, J. Davy Kirkpatrick ${ }^{2}$, Christopher R. Gelino ${ }^{2,3}$, Gregory N. Mace ${ }^{4,5}$, \\ Edward L. Wright ${ }^{4}$, Peter R. Eisenhardt ${ }^{6}$, M. F. Skrutskie ${ }^{7}$, Roger L. Griffith ${ }^{8}$, And Kenneth A. Marsh ${ }^{9}$ \\ ${ }^{1}$ Department of Physics and Astronomy, University of Toledo, 2801 W. Bancroft St., Toledo, OH 43606, USA; Adam.Schneider@Utoledo.edu \\ ${ }^{2}$ Infrared Processing and Analysis Center, MS 100-22, California Institute of Technology, Pasadena, CA 91125, USA \\ ${ }^{3}$ NASA Exoplanet Science Institute, Mail Code 100-22, California Institute of Technology, 770 South Wilson Ave., Pasadena, CA 91125, USA \\ ${ }_{5}^{4}$ Department of Physics and Astronomy, UCLA, 430 Portola Plaza, Box 951547, Los Angeles, CA 90095-1547, USA \\ ${ }^{5}$ Department of Astronomy, The University of Texas at Austin, 2515 Speedway, Stop C1400, Austin, TX 78712, USA \\ 6 Jet Propulsion Laboratory, California Institute of Technology, 4800 Oak Grove Dr., Pasadena, CA 91109, USA \\ 7 Department of Astronomy, University of Virginia, 530 McCormick Road, Charlottesville, VA 22904, USA \\ ${ }^{8}$ Department of Astronomy \& Astrophysics, 525 Davey Lab, The Pennsylvania State University, University Park, PA 16802, USA \\ ${ }^{9}$ School of Physics and Astronomy, Cardiff University, Cardiff CF24 3AA, UK \\ Received 2014 October 2; accepted 2015 February 18; published 2015 May 6
}

\begin{abstract}
We present a sample of brown dwarfs identified with the Wide-field Infrared Survey Explorer (WISE) for which we have obtained Hubble Space Telescope (HST) Wide Field Camera 3 (WFC3) near-infrared grism spectroscopy. The sample (22 in total) was observed with the G141 grism covering 1.10-1.70 $\mu \mathrm{m}$, while 15 were also observed with the G102 grism, which covers $0.90-1.10 \mu \mathrm{m}$. The additional wavelength coverage provided by the G102 grism allows us to (1) search for spectroscopic features predicted to emerge at low effective temperatures (e.g., ammonia bands) and (2) construct a smooth spectral sequence across the T/Y boundary. We find no evidence of absorption due to ammonia in the G102 spectra. Six of these brown dwarfs are new discoveries, three of which are found to have spectral types of T8 or T9. The remaining three, WISE J082507.35+280548.5 (Y0.5), WISE J120604.38+840110.6 (Y0), and WISE J235402.77+024015.0 (Y1), are the 19th, 20th, and 21st spectroscopically confirmed Y dwarfs to date. We also present $H S T$ grism spectroscopy and reevaluate the spectral types of five brown dwarfs for which spectral types have been determined previously using other instruments.
\end{abstract}

Key words: stars: low-mass - brown dwarfs

Supporting material: data behind figures

\section{INTRODUCTION}

The Wide-field Infrared Survey Explorer (WISE) has been very successful at identifying the coolest brown dwarfs in the solar neighborhood. WISE provided all sky coverage at four mid-infrared wavelengths centered at 3.4, 4.6, 12 and $22 \mu \mathrm{m}$ $(W 1, W 2, W 3$, and $W 4)$. This coverage has offered the ideal data set with which to identify cool brown dwarfs whose spectral energy distributions peak at mid-infrared wavelengths. Specifically, WISE was designed so that the $W 1$ band coincides with a deep water+methane absorption feature and the $W 2$ band coincides with a region largely free of opacity. Thus, the WISE $W 1-W 2$ color has been especially useful for identifying latetype dwarfs (Kirkpatrick et al. 2011; Cushing et al. 2011; Kirkpatrick et al. 2012; Mace et al. 2013; Thompson et al. 2013). Seventeen of the 18 spectroscopically confirmed brown dwarfs with spectral types of $\mathrm{Y0}$ or later were first identified by WISE (Cushing et al. 2011, 2014a; Kirkpatrick et al. 2012, 2013; Tinney et al. 2012; Pinfield et al. 2014). The Y0 dwarf WISE J1217+16 B was identified as a companion to a T8.5 dwarf, itself identified by WISE (Liu et al. 2012; Leggett et al. 2015). Three objects, WD 0806-661(Luhman et al. 2011), CFBDSIR J1458+1013 B (Liu et al. 2011), and WISE 0855-0714 (Luhman 2014), likely have effective temperatures similar to (or less than) the above Y dwarfs, but have yet to be spectroscopically confirmed.

Ground-based follow-up observations at the mid-infrared wavelengths where the spectral energy distributions of cold brown dwarfs peak are nearly impossible due to the high thermal background, forcing follow-up observations to shorter wavelengths where these dwarfs are extremely faint. As a result, obtaining moderate signal-to-noise $(\mathrm{S} / \mathrm{N})$ near-infrared spectra for many of the coldest WISE candidates has only been capable with the Wide Field Camera 3 (WFC3) aboard the Hubble Space Telescope (HST). HST grism spectroscopy of the latest-type brown dwarfs has been invaluable in the study and classification of these objects (Cushing et al. 2011, 2014a; Kirkpatrick et al. 2012, 2013).

As effective temperatures cool below $600 \mathrm{~K}$, several spectroscopic features are predicted by model atmospheres to arise in the Y-band spectral region around $1.07 \mu \mathrm{m}$. These include the emergence of ammonia absorption components and the disappearance of optical alkali resonance lines, which have broad wings that are predicted to extend into the near-infrared (Burrows et al. 2000; Kirkpatrick et al. 2012). (HST) WFC3 G102 spectroscopy $(0.90-1.10 \mu \mathrm{m})$ allows us to investigate this additional wavelength range for such features, as well as inspect for differences (and similarities) as a function of spectral type.

In this paper, we present our HST brown dwarf spectroscopic sample, including three new Y-dwarfs: WISEA J082507.37 +280548.2 , WISEA J120604.25 + 840110.5, and WISEA J235402.79+024014.1. We also present improved (higher S/ N) HST spectroscopy of five additional brown dwarfs for which near-infrared spectroscopy has been published previously, and we present $H S T$ G102 grism spectroscopy for a sample of 15 late $\mathrm{T}$ and $\mathrm{Y}$ dwarfs. We first present the six new WISE brown dwarf discoveries and then present the new HST 
Table 1

AllWISE Photometry

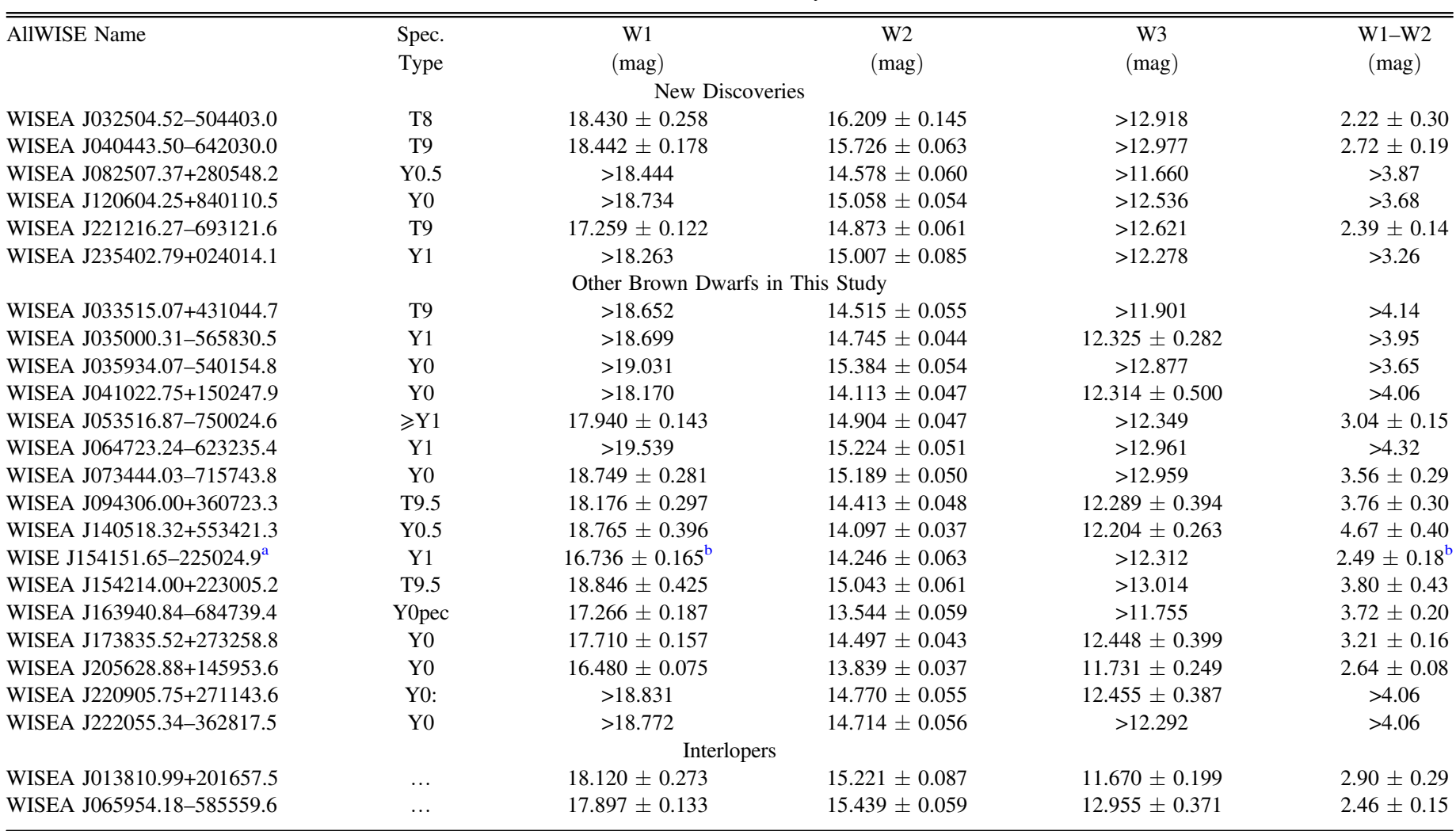

${ }^{\text {a }}$ WISE $1541-2250$ is not in the AllWISE catalog.

b Details of the likely erroneous W1 measurement of WISE 1541-2250 are discussed in Kirkpatrick et al. (2012).

WFC3 spectroscopy of previously identified brown dwarfs. We then present and analyze the Y-band spectra for the 15 brown dwarfs observed with the G102 grism and construct a complete spectral sequence across the T/Y boundary. Lastly, we estimate physical properties of our entire sample of brown dwarfs by atmospheric model fitting.

\section{THE SAMPLE}

The selection criteria for brown dwarfs in this study are described in detail in Kirkpatrick et al. (2012). AllWISE source catalog positions and photometry for all 22 brown dwarfs in this study, including the six new discoveries, are given in Table 1. (Note: a detailed analysis of the HST spectrum of WISEA J182831.08+265037.6 will be published separately in Cushing et al., in preparation.) We provide data from the AllWISE catalog because of its improved astrometric accuracy and increased sensitivity in the W1 and W2 bands compared to the WISE All-Sky catalog. An in-depth characterization of the AllWISE source catalog can be found in Kirkpatrick et al. (2014) and the AllWISE Explanatory Supplement ${ }^{10}$. Hereafter, source names are abbreviated using the first four digits of the right ascension and declination for each object (e.g., WISEA J120604.25+840110.5 is WISE $1206+8401)$. Finder charts for each of the new discoveries are presented in Figures 1-6.

\footnotetext{
${ }^{10}$ See http://wise2.ipac.caltech.edu/docs/release/allwise/expsup/
}

\section{OBSERVATIONS}

\subsection{Photometry}

\subsubsection{WFC3/HST}

Direct images of each brown dwarf were obtained with the Wide Field Camera 3 (WFC3; Kimble et al. 2008) aboard the Hubble Space Telescope. Each grism observation (Section 3.2) requires an accompanying direct image, necessary for locating sources and determining source sizes. Positions and source sizes are then used to determine the placement and size of the corresponding grism extraction apertures and the wavelength zero point. These images can also be used for photometric purposes. Direct images for the G141 grism observations were obtained with either the F140W $\left(\lambda_{p}=1392.3 \mathrm{~nm}\right)$ or F125W filters $\left(\lambda_{p}=1248.6 \mathrm{~nm}\right)$, while images for the G102 grism observations were obtained with the F105W filter $\left(\lambda_{p}=1055.2\right.$ $\mathrm{nm}$ ), where $\lambda_{p}$ is the "pivot wavelength" (see Tokunaga \& Vacca 2005). The $1024 \times 1024 \mathrm{HgCdTe}$ detector used by WFC3 has a plate scale of $\sim 00^{\prime \prime} 13$ per pixel, resulting in a total field of view of $123^{\prime \prime} \times 126^{\prime \prime}$. Multiple images were obtained for each source and were combined using AstroDrizzle (Gonzaga et al. 2012).

To test the consistency of our measured magnitudes, we performed aperture photometry on both the individual flat-field frames ( $f t$-multiplied by the WFC3 IR pixel area map to account for geometric distortion) and the final drizzled image created by AstroDrizzle $(d r z)$. In several instances, we found differences between the magnitudes derived from the $f t$ and $d r z$ images of up to $\sim 0.2 \mathrm{mag}$, similar to the results of Kirkpatrick 


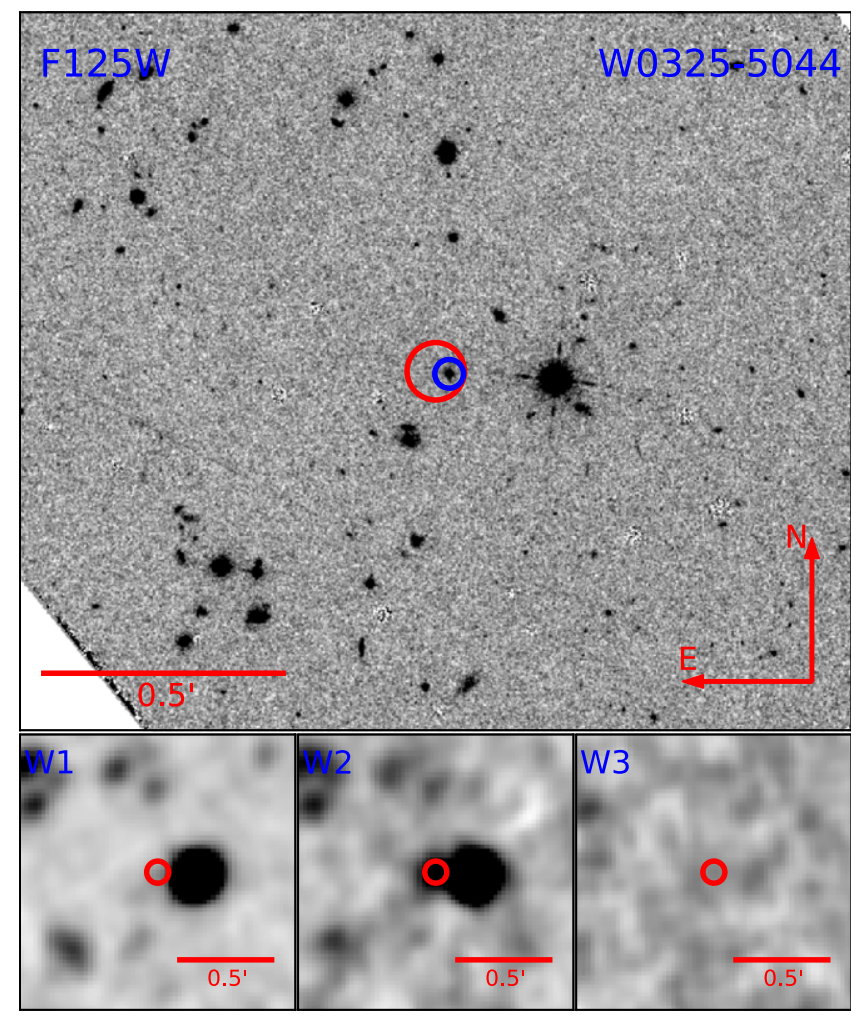

Figure 1. Finder chart for new brown dwarf discovery WISE 0325-5044. The top panel is the HST WFC3 F125W image, while the bottom three panels are WISE channels 1-3, from left to right. All panels are centered on the WISE position of the brown dwarf, which is also indicated by a red circle. In the top panel, the HST brown dwarf position is indicated by a blue circle. North is up and East is left in each panel.

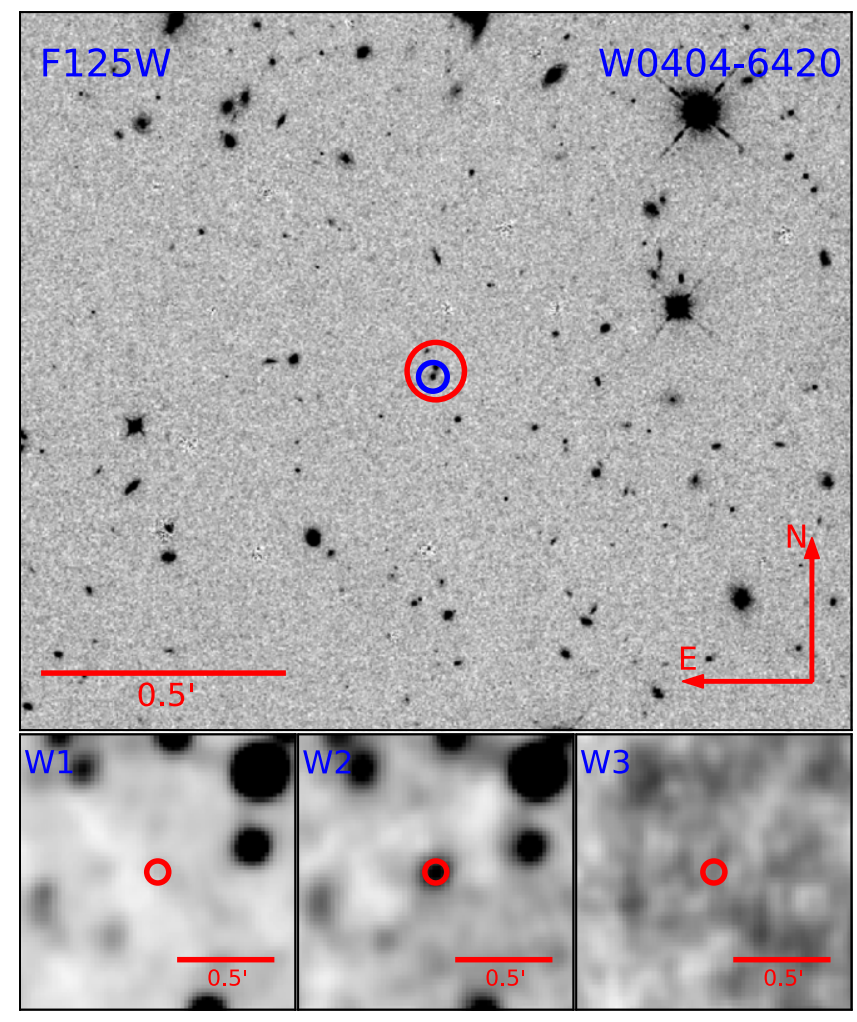

Figure 2. Same as Figure 1, but for WISE 0404-6420.

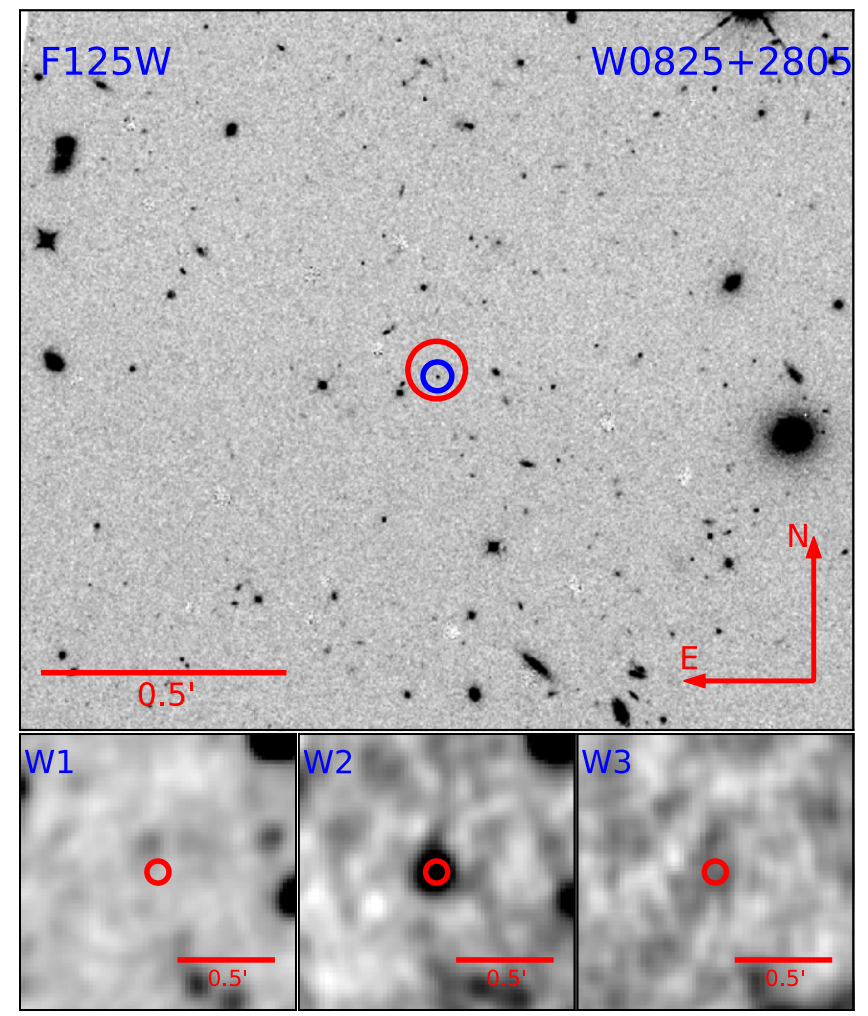

Figure 3. Same as Figure 1, but for WISE $0825+2805$.

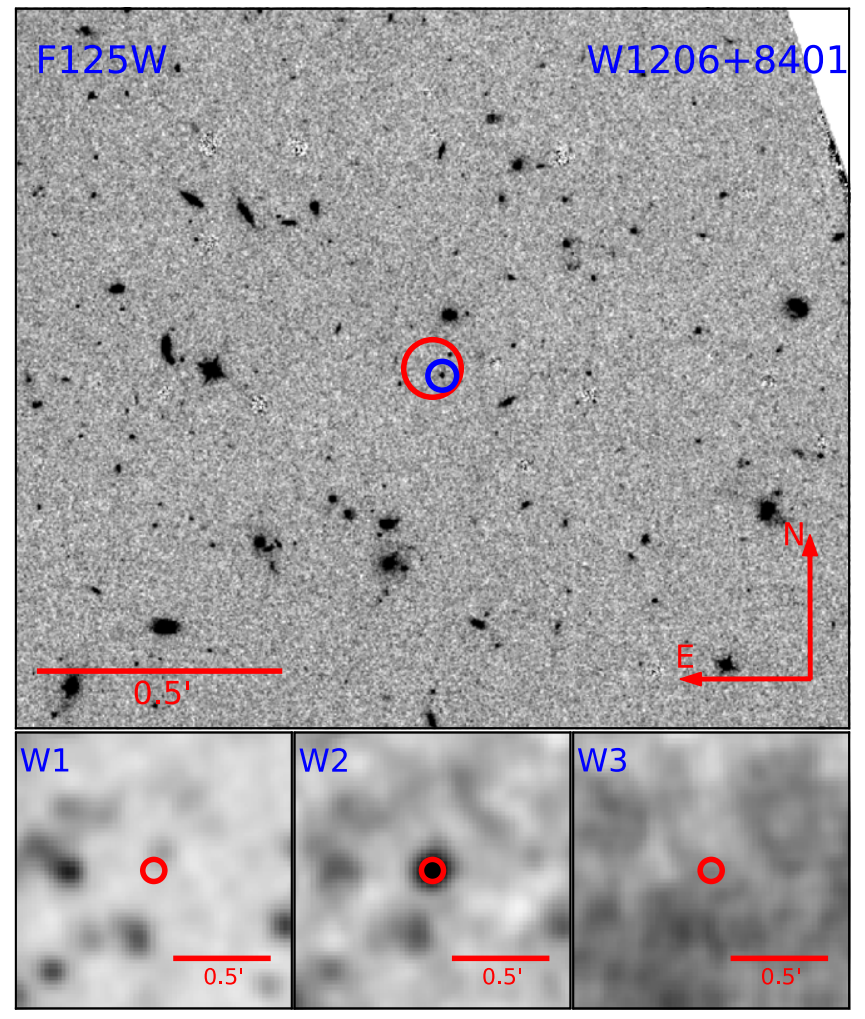

Figure 4. Same as Figure 1, but for WISE 1206+8401.

et al. (2012). Further investigation revealed the cause of these discrepancies to be liberal default values for the cosmic-ray rejection algorithm of AstroDrizzle. On occasion, this algorithm will flag a central pixel of a source as a cosmic ray, thus 


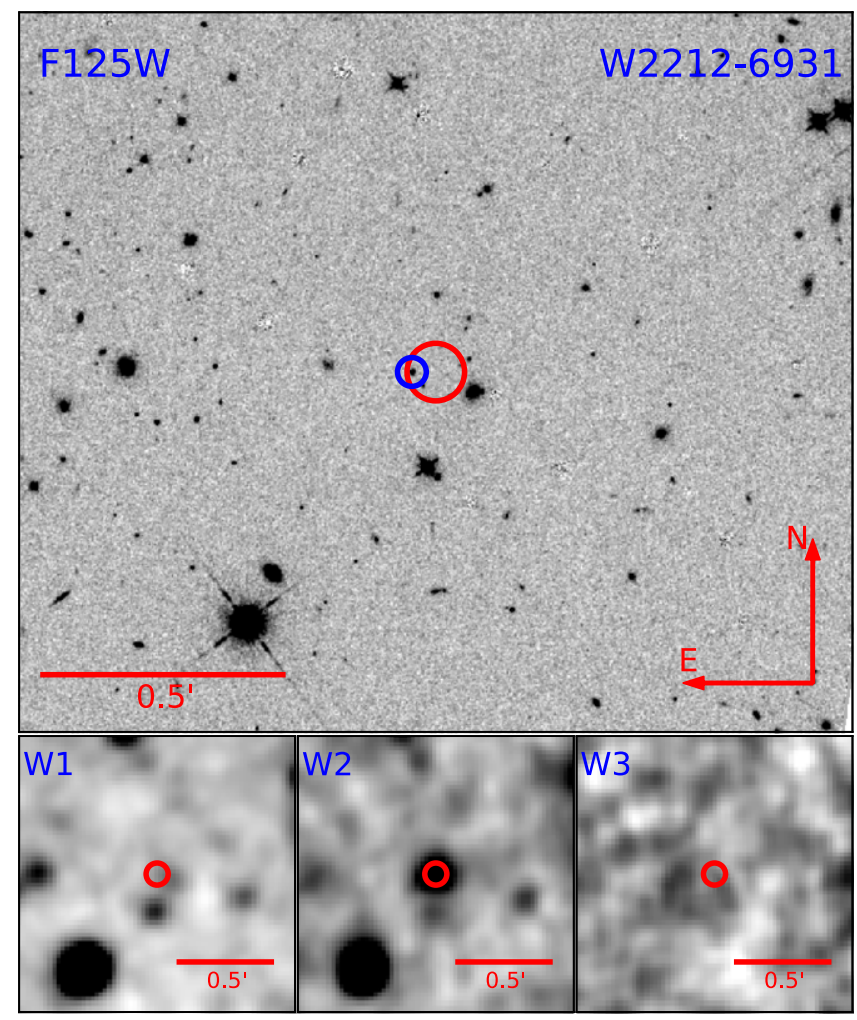

Figure 5. Same as Figure 1, but for WISE 2212-6931.

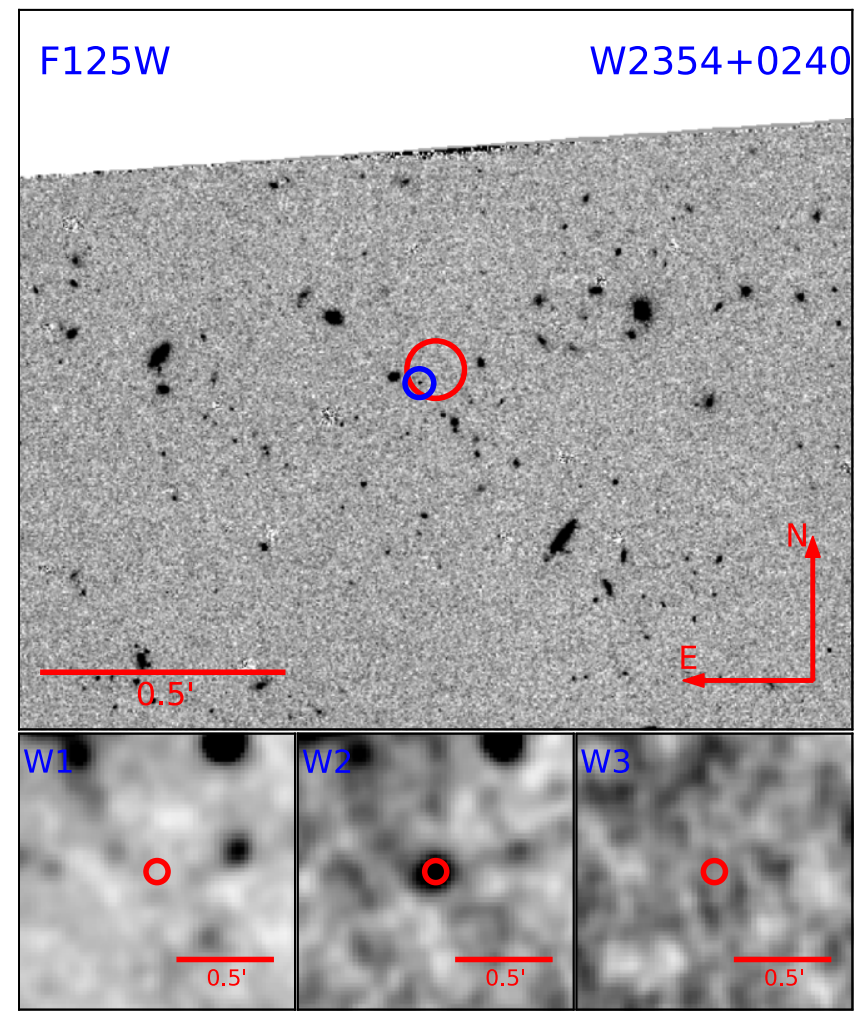

Figure 6. Same as Figure 1, but for WISE 2354+0240.

reducing the total flux in the combined final $d r z$ image compared to the original $f t$ images. Because cosmic rays are rejected in the calwf 3 calibration program used to create the $f t$ images, we corrected this issue by first turning off the cosmic- ray rejection algorithm when combining the $f t$ frames with AstroDrizzle. The $d r z$ images created by AstroDrizzle in this way were found to contain several artifacts (not cosmic rays) that are not present in the $d r z$ images created by AstroDrizzle when the cosmic-ray rejection algorithm is turned on. We remove these artifacts by employing a Laplacian edge detection algorithm (van Dokkum 2001) to the $d r z$ images created by AstroDrizzle with the cosmic-ray rejection algorithm turned off. Aperture photometry is then performed on these final images.

One result of the drizzling process is that the noise in adjacent pixels is correlated. For this reason, determining the rms noise in the local background using common background subtraction methods, such as a sky annulus, is inapplicable. Instead, we estimate the background and its uncertainty by applying the same aperture used for the source photometry $(0.4)$ to 1000 random star-free positions (determined via a $3 \sigma$ clip) on each image. We take the mean and standard deviation of these measurements as the background level and its uncertainty. This uncertainty is added in quadrature to the signal uncertainty, determined by dividing source counts by the gain and total exposure time, to determine the final source uncertainty. Magnitudes were calculated using the Vega system with zero points of $25.1845,25.1439$, and 25.4523 for F140W, F125W, and F105W images, respectively. ${ }^{11} H S T$ photometric magnitudes and uncertainties are given in Table 2.

\subsubsection{IRAC/Spitzer}

The entire sample was observed with the InfraRed Array Camera (IRAC; Fazio et al. 2004) aboard the Spitzer Space Telescope. Each dwarf was imaged with IRAC channels 1 and 2 centered at 3.6 and $4.5 \mu \mathrm{m}$ (hereafter, chl and ch2). A detailed description of the IRAC data reduction procedure used to determine chl and ch2 magnitudes is given in Kirkpatrick et al. (2011). ch1 and ch2 magnitudes for each dwarf are given in Table 2. For WISE 1639-6847, which was blended with another source in its chl image, only a ch2 magnitude is given.

\subsection{Spectroscopy}

HST spectroscopic observations were carried out using Wide Field Camera 3 (WFC3) as part of Cycle 18 program 12330 (PI: Kirkpatrick), Cycle 19 program 12544 (PI: Cushing), and Cycle 20 programs 12970 (PI: Cushing) and 13178 (PI: Kirkpatrick) and are summarized in Table 3. The G141 $(1.1-1.7 \mu \mathrm{m}, \quad R \equiv \lambda / \Delta \lambda \approx 130)$ and G102 (0.9-1.1 $\mu \mathrm{m}$, $R \approx 210$ ) spectroscopic reductions were performed using the methods described in Cushing et al. (2011), with the exception of one final step, described in the following paragraphs.

Because the G141 and G102 grism modes are slitless, source spectra are occasionally influenced by photons from a nearby source or uneven background fluctuations. For this reason, we developed a custom source extraction routine that allows us to define source apertures and background regions on the individual stamp images of a spectrum produced with the AXEDRIZZLE routine (e.g., Figure 7). Once an aperture is defined, we use the aperture corrections of Kuntschner et al. (2011) and the appropriate grism sensitivity curves $^{12}$ to fluxcalibrate the final spectrum. There is also a $2 \%$ absolute flux calibration uncertainty (Kuntschner et al. 2011), which we

\footnotetext{
11 http://stsci.edu/hst/wfc3/phot_zp_lbn

$12 \mathrm{http} / / /$ stsci.edu/hst/wfc3/analysis/grism_obs/wfc3-grism-resources.html
} 
Table 2

$H S T$ and Spitzer Aperture Photometry

\begin{tabular}{|c|c|c|c|c|c|c|}
\hline AllWISE Name & $\begin{array}{c}\text { F105W } \\
\text { (mag) }\end{array}$ & $\begin{array}{c}\text { F125W } \\
\text { (mag) }\end{array}$ & $\begin{array}{c}\text { F140W } \\
\text { (mag) }\end{array}$ & $\begin{array}{c}\text { chl } \\
(\mathrm{mag})\end{array}$ & $\begin{array}{c}\text { ch2 } \\
(\mathrm{mag})\end{array}$ & $\begin{array}{c}\text { ch1-ch2 } \\
(\mathrm{mag})\end{array}$ \\
\hline WISEA J032504.52-504403.0 & $20.618 \pm 0.009$ & $19.598 \pm 0.002$ & $\ldots$ & $17.746 \pm 0.086$ & $15.696 \pm 0.025$ & $2.050 \pm 0.090$ \\
\hline WISEA J033515.07+431044.7 & $20.880 \pm 0.015$ & $20.092 \pm 0.003$ & $\ldots$ & $16.612 \pm 0.040$ & $14.381 \pm 0.020$ & $2.231 \pm 0.045$ \\
\hline WISEA J035000.31-565830.5 & $\ldots$ & $\ldots$ & $22.321 \pm 0.047$ & $17.936 \pm 0.096$ & $14.688 \pm 0.020$ & $3.248 \pm 0.098$ \\
\hline WISEA J035934.07-540154.8 & $\ldots$ & $\ldots$ & $21.806 \pm 0.039$ & $17.553 \pm 0.072$ & $15.326 \pm 0.023$ & $2.227 \pm 0.075$ \\
\hline WISEA J040443.50-642030.0 & $21.115 \pm 0.013$ & $20.276 \pm 0.003$ & $\ldots$ & $17.633 \pm 0.082$ & $15.418 \pm 0.022$ & $2.216 \pm 0.085$ \\
\hline WISEA J041022.75+150247.9 & $\ldots$ & $\ldots$ & $19.634 \pm 0.007$ & $16.636 \pm 0.042$ & $14.166 \pm 0.019$ & $2.470 \pm 0.046$ \\
\hline WISEA J053516.87-750024.6 & $23.140 \pm 0.049$ & $22.801 \pm 0.051$ & $22.422 \pm 0.050$ & $17.753 \pm 0.084$ & $15.009 \pm 0.021$ & $2.744 \pm 0.087$ \\
\hline WISEA J064723.24-623235.4 & $23.592 \pm 0.054$ & $23.453 \pm 0.050$ & $\ldots$ & $17.893 \pm 0.092$ & $15.070 \pm 0.022$ & $2.823 \pm 0.094$ \\
\hline WISEA J073444.03-715743.8 & $21.732 \pm 0.031$ & $20.964 \pm 0.004$ & $\ldots$ & $17.649 \pm 0.077$ & $15.213 \pm 0.022$ & $2.436 \pm 0.080$ \\
\hline WISEA J082507.37+280548.2 & $23.487 \pm 0.040$ & $23.197 \pm 0.030$ & $\ldots$ & $17.624 \pm 0.077$ & $14.637 \pm 0.020$ & $2.987 \pm 0.079$ \\
\hline WISEA J094306.00+360723.3 & $\ldots$ & $\ldots$ & $20.037 \pm 0.007$ & $16.746 \pm 0.043$ & $14.284 \pm 0.019$ & $2.461 \pm 0.047$ \\
\hline WISEA J120604.25+840110.5 & $21.694 \pm 0.025$ & $21.062 \pm 0.005$ & $\ldots$ & $17.339 \pm 0.061$ & $15.220 \pm 0.022$ & $2.119 \pm 0.065$ \\
\hline WISEA J140518.32+553421.3 & $21.939 \pm 0.024$ & $\ldots$ & $21.271 \pm 0.018$ & $16.876 \pm 0.046$ & $14.058 \pm 0.019$ & $2.818 \pm 0.050$ \\
\hline WISE J154151.65-225024.9 & $22.204 \pm 0.044$ & $21.871 \pm 0.023$ & $\ldots$ & $16.658 \pm 0.042$ & $14.228 \pm 0.019$ & $2.430 \pm 0.046$ \\
\hline WISEA J154214.00+223005.2 & $21.268 \pm 0.020$ & $20.630 \pm 0.003$ & $20.240 \pm 0.010$ & $17.257 \pm 0.059$ & $15.057 \pm 0.022$ & $2.200 \pm 0.062$ \\
\hline WISEA J163940.84-684739.4 & $21.337 \pm 0.026$ & $21.151 \pm 0.012$ & $\ldots$ & $\ldots$ & $13.537 \pm 0.017$ & \\
\hline WISEA J173835.52+273258.8 & $\ldots$ & $\ldots$ & $19.883 \pm 0.008$ & $17.093 \pm 0.053$ & $14.473 \pm 0.019$ & $2.620 \pm 0.056$ \\
\hline WISEA J205628.88+145953.6 & $\ldots$ & $\ldots$ & $19.524 \pm 0.007$ & $16.031 \pm 0.030$ & $13.923 \pm 0.018$ & $2.108 \pm 0.035$ \\
\hline WISEA J220905.75+271143.6 & $23.842 \pm 0.057$ & $\ldots$ & $23.167 \pm 0.149$ & $17.815 \pm 0.087$ & $14.739 \pm 0.020$ & $3.076 \pm 0.090$ \\
\hline WISEA J221216.27-693121.6 & $21.069 \pm 0.012$ & $20.347 \pm 0.003$ & $\ldots$ & $17.364 \pm 0.063$ & $14.973 \pm 0.021$ & $2.391 \pm 0.066$ \\
\hline WISEA J222055.34-362817.5 & $21.638 \pm 0.027$ & $20.997 \pm 0.005$ & $\ldots$ & $17.200 \pm 0.057$ & $14.736 \pm 0.021$ & $2.464 \pm 0.061$ \\
\hline WISEA J235402.79+024014.1 & $\ldots$ & $23.368 \pm 0.094$ & $\ldots$ & $18.105 \pm 0.109$ & $15.013 \pm 0.022$ & $3.091 \pm 0.111$ \\
\hline
\end{tabular}

account for in all synthetic photometry calculations (Section 5.3) and model fits (Section 5.4). An example of the functionality of our extraction technique is shown in Figure 7, where the effects of uneven background fluctuations are mitigated.

Several sources had multiple $H S T$ visits (i.e., spectroscopic observations occurring on different dates). In principle, direct and spectroscopic images from multiple visits can be combined using standard AstroDrizzle routines to produce final images with improved S/N. Yet, because our sources are nearby brown dwarfs, they tend to have significant proper motions, even on timescales as short as a month. For HST grism observations, identical positions are critical for the placement of extraction apertures and wavelength zero points. For sources with multiple visits, AXEDRIZZLE stamp images from each visit are median combined to produce a final spectroscopic image. Spectra are then extracted using the method described above. Objects with both G141 and G102 data are stitched together at $1.1 \mu \mathrm{m}$ with no scaling done between the two spectra. The complete spectroscopic sample is shown in Figures 8 and 9. We note here that the depressed H-band peak of WISE 0647-6232 seen in Kirkpatrick et al. (2013) does not appear in our reduced spectrum. This is likely due to either the added signal of our spectrum or our improved spectral extraction technique. WISE 0535-7500 is located in an extremely crowded field, and thus retrieving a clean G102 spectrum was particularly difficult, even with multiple observations at multiple roll angles. While we did manage to extract a G102 spectrum for this object, we urge caution in its interpretation, as it is still likely contaminated. We also note that a different roll angle allowed us to extract a cleaner J-band spectrum than that found in Kirkpatrick et al. (2012), though the zeroth-order light from a nearby object fell directly in the H-band portion of WISE 0535-7500's spectrum. For this reason, we do not use the H-band portion of this object's spectrum.

Upon inspection of their spectra, two objects that met the selection criteria were found to not be brown dwarfs. The spectra for both of these objects were flat and devoid of spectroscopic features and are likely extragalactic in nature. The AllWISE positions and photometry for these contaminants are provided in Table 1.

\section{ANALYSIS}

\subsection{New Discoveries}

Spectral types for the six new brown dwarf discoveries (WISE 0325-5044, WISE 0404-6420, WISE 0825+2805, WISE 1206 +8401, WISE 2212-6931, and WISE 2354+0240) were determined following the methods of Cushing et al. (2011) and Kirkpatrick et al. (2012). These discoveries are classified via byeye comparisons of the width of the J-band peak to the spectral standards defined in Burgasser et al. (2006); Cushing et al. (2011), and Kirkpatrick et al. (2013). Comparisons are shown in Figures 8 and 9, while the spectral types are provided in Table 1.

The spectrum of WISE 0325-5044 is an excellent match to the T8 spectral standard 2MASSI J0415195-093506 (Burgasser et al. 2006); hence, we classify it as a T8. Similarly, WISE 0404-6420 and WISE 2212-6931 show excellent agreement with the T9 spectral standard UGPS J072227.51-054031.2 (Cushing et al. 2011) and are classified as such. WISE 1206 +8401 is an almost perfect match to the Y0 spectral standard WISE J173835.53+273259.0 (Cushing et al. 2011); therefore, we are confident in assigning it a Y0 spectral classification. Even though the spectrum of WISE 2354+0240 is somewhat noisy, it shows a J-band peak significantly narrower than the Y0 spectral standard and matches well with the Y1 standard WISE J035000.32-565830.2 (Kirkpatrick et al. 2012). Therefore, we assign a spectral type of $\mathrm{Y} 1$ for this object, making it the fourth such object to receive such a designation, along with WISE J035000.32-565830.2 (Kirkpatrick et al. 2012), WISE J064723.23-623235.5 (Kirkpatrick et al. 2013), and WISE J154151.65-225024.9 (see Section 4.2). The most ambiguous of all classifications was that of WISE $0825+2805$, which is shown separately in Figure 10. A comparison of WISE 0825 
Table 3

HST/WFC3 Spectroscopy Log

\begin{tabular}{|c|c|c|c|c|}
\hline AllWISE Name & $\begin{array}{l}\text { Date } \\
\text { (UT) }\end{array}$ & Mode & $\begin{array}{r}\text { Total Int.Time } \\
(\mathrm{s})\end{array}$ & \# of Exp. \\
\hline \multirow[t]{2}{*}{ WISEA J032504.52-504403.0 } & 2013 July 30 & G102 & 1612 & \\
\hline & 2013 Aug 4 & G141 & 1612 & \\
\hline & 2013 Aug 30 & G141 & 1812 & \\
\hline WISEA J035000.31-565830.5 & 2011 Aug 13 & G141 & 2212 & \\
\hline WISEA J035934.07-540154.8 & 2011 Aug 10 & G141 & 2212 & \\
\hline WISEA J041022.75+150247.9 & 2012 Sep 1 & G141 & 2012 & \\
\hline \multirow[t]{5}{*}{ WISEA J053516.87-750024.6 } & 2011 Sep 27 & G141 & 2212 & \\
\hline & 2012 Sep 17 & G141 & 2212 & \\
\hline & 2013 Sep 26 & G102 & 7618 & \\
\hline & 2013 Sep 27 & G141 & 7615 & \\
\hline & 2013 Sep 27 & G102 & 7618 & \\
\hline & 2013 Nov 14 & G102 & 7036 & 12 \\
\hline & 2013 Dec 28 & G102 & 7036 & 12 \\
\hline & 2013 Dec 29 & G141 & 7218 & 6 \\
\hline & 2013 Dec 30 & G102 & 7036 & 12 \\
\hline \multirow[t]{2}{*}{ WISEA J073444.03-715743.8 } & 2013 May 18 & G102 & 1812 & 4 \\
\hline & 2013 May 20 & G141 & 1812 & 4 \\
\hline \multirow[t]{4}{*}{ WISEA J082507.37+280548.2 } & 2014 Jan 16 & G102 & 7018 & \\
\hline & 2014 Jan 17 & G141 & 7218 & \\
\hline & 2014 Jan 18 & G102 & 7018 & \\
\hline & 2014 Jan 19 & G102 & 7018 & \\
\hline WISEA J094306.00+360723.3 & 2013 Feb 20 & G141 & 2012 & \\
\hline \multirow[t]{2}{*}{ WISEA J120604.25+840110.5 } & 2013 July 15 & G102 & 1812 & \\
\hline & 2013 July 15 & G141 & 1812 & 4 \\
\hline & 2013 Oct 27 & G102 & 7518 & \\
\hline & 2013 Oct 29 & G141 & 10024 & 4 \\
\hline WISEA J173835.52+273258. $8^{\mathrm{c}}$ & 2011 May 12 & G141 & 2012 & \\
\hline WISEA J205628.88+145953.6 ${ }^{\mathrm{a}}$ & 2011 Sept 4 & G141 & 2012 & 4 \\
\hline \multirow[t]{4}{*}{ WISEA J220905.75+271143.6 ${ }^{\mathrm{d}}$} & 2012 Sept 15 & G141 & 2012 & 4 \\
\hline & 2013 Apr 28 & G102 & 7218 & \\
\hline & 2013 June 6 & G102 & 7218 & \\
\hline & 2013 Sept 20 & G102 & 7218 & \\
\hline \multirow[t]{2}{*}{ WISEA J221216.27-693121.6 } & 2013 Sept 7 & G102 & 4612 & \\
\hline & 2013 Sept 11 & G141 & 1812 & \\
\hline \multirow[t]{2}{*}{ WISEA J222055.34-362817.5 } & 2013 June 8 & G102 & 1812 & \\
\hline & 2013 June 20 & G141 & 4412 & 4 \\
\hline WISEA J235402.79+024014.1 & 2013 Sept 22 & G141 & 3224 & 8 \\
\hline
\end{tabular}

Notes:

a Published previously in Kirkpatrick et al. (2012).

b Published previously in Kirkpatrick et al. (2013).

${ }^{\mathrm{c}}$ Published previously in Cushing et al. (2011).

${ }^{\mathrm{d}}$ Published previously in Cushing et al. (2014a).

+2805 with the Y0 and Y1 spectral standards shows that the width of the J-band peak for this object is intermediate between the two. For this reason we assign WISE $0825+2805$ a spectral type of Y0.5.
While all of these discoveries are part of an extensive parallax program to measure their distances, we provide preliminary estimates here based solely on W2 photometry and the polynomial fits from Dupuy \& Liu (2012) and 

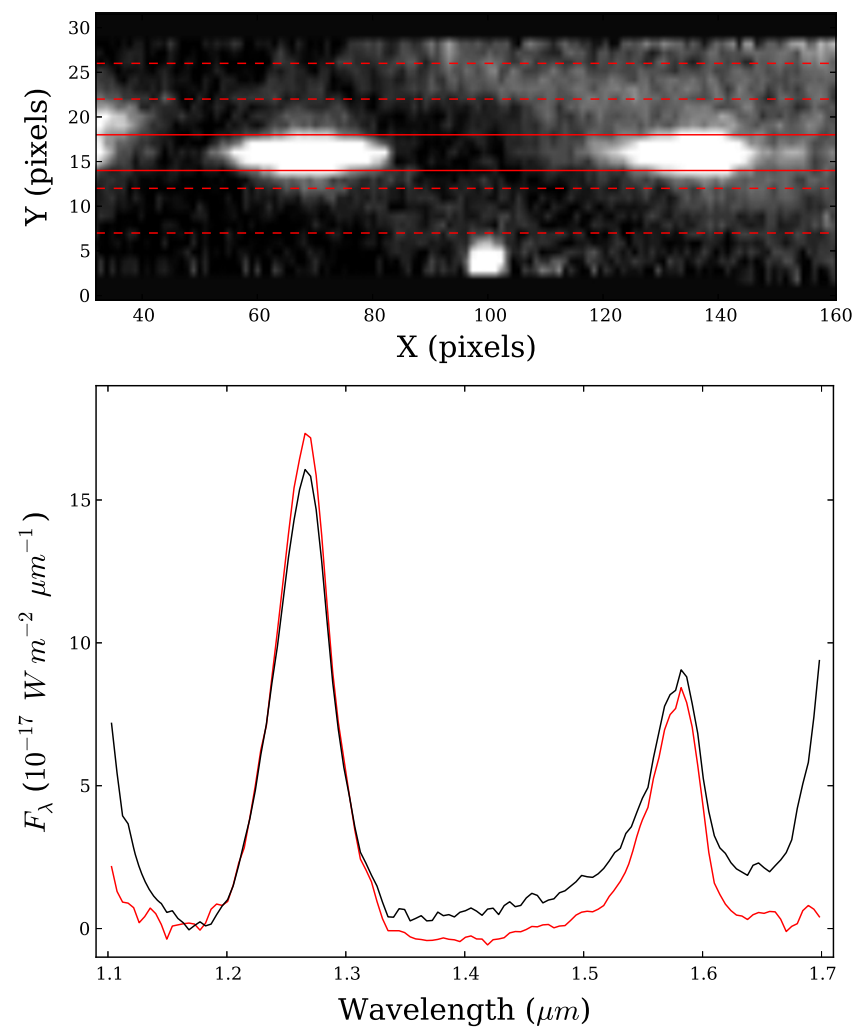

Figure 7. Top: Stamp image of G141, the grism spectrum of WISE 2056 +1459 . Solid red lines indicate the defined extraction aperture. Dashed lines show the regions used for background fitting/subtraction. Bottom: Comparison of the AXEDRIZZLE extracted spectrum of WISE 2056+1459 (black) and the spectrum extracted with our routine (red).

Kirkpatrick et al. (2012). Distances estimates are provide in Table 4. Uncertainties are based solely on the photometric uncertainty of the W2 measurement.

\subsection{Improved Spectroscopy of Previously Identified Brown Dwarfs}

Figures 8 and 9 also show HST spectroscopy of five previously known brown dwarfs, including WISE J0335+4310 (T9-Mace et al. 2013), WISE J07344-7157 (Y0-Kirkpatrick et al. 2012), WISE J1541-2250 ("Y0.5?"-Kirkpatrick et al. 2012), WISE J1639-6847 (Y0-Y0.5-Tinney et al. 2012), and WISE J2220-3628 (Y0-Kirkpatrick et al. 2012). Spectral types were reexamined using the methods described in Section 4.1. Spectral types determined in this way agree well with the spectral types determined in Mace et al. (2013); Kirkpatrick et al. (2012), and Tinney et al. (2012). With the improved S/N from HST, we reclassify W1541-2250 as $\mathrm{Y} 1$ because the width of its J-band peak is a much better match to the Y1 standard than that of the Y0 standard. This determination supersedes the "Y0.5?" suggested in Kirkpatrick et al. (2012). The J-band peak of WISE 1639-6847 matches well with the Y0 standard, in agreement with the Y0-Y0.5 spectral type determined in Tinney et al. (2012). However, the Y-band peak and $Y-J$ color of this object are unusual compared to other Y0 dwarfs (see Section 5.1 and Figure 13); therefore, we classify WISE 1639-6847 as Y0pec.

\section{DISCUSSION}

\subsection{Spectral Sequence}

In Figure 11 we show the entire combined HST G102/G141 near-infrared spectra for a complete sequence of late-type brown dwarfs from T8 to Y1. There is a distinct trend for the Y-, J-, and H-band peaks as temperatures decrease. The Y-band peak appears more symmetrical going from T8 to Y1. This occurs because the blue wing of the Y-band peak becomes more suppressed for later spectral types. The wavelength at which the peak reaches a maximum also occurs at a different location as temperature decreases, gradually becoming bluer with spectral type. The J-band peak becomes narrower as temperatures decrease, while the H-band peak appears to become more symmetrical for later spectral types. The peaks of both the $\mathrm{J}$ and $\mathrm{H}$ bands occur at the same position throughout the sequence.

G102 observations were made for five of the six new brown dwarfs presented in Section 4.1 (the exception being WISE 2354+0240), all five dwarfs presented in Section 4.2, and five additional brown dwarfs, including WISE 0535-7500 ( $\geqslant$ Y1Kirkpatrick et al. 2012), WISE 0647-6232 (Y1-Kirkpatrick et al. 2013), WISE 1405+5534 (Y0pec-Cushing et al. 2011), WISE 1542+2230 (T9.5-Mace et al. 2013), and WISE $2209+2711$ (Y0:-Cushing et al. 2014a). The G102 spectra are presented in Figure 12.

WISE 1639-6847 shows an increase in the relative height of the Y-band peak compared to other Y0 type dwarfs. The peak is also shifted blueward from the peak of the T9 standard UGPS J072227.51-054031.2. This is a surprising result considering that we find that the J-band peak matches well with the Y0 standard (see Figure 9). Because the Y-band shape of this object is so unusual, we classify it as a Y0pec. We note that a similarly large Y-band peak is seen in the FIRE spectrum of WISE 1639-6847 presented in Tinney et al. (2012) (see their Figure 7).

The Y0pec dwarf WISE 1405+5534 (which is categorized as "pec" due to a wavelength shift of its H-band peak) shows a Yband peak slightly different from that of the T9 and Y0 dwarfs. Though noisy, the Y-band peak of WISE 1405+5534 is more symmetrical about its maximum than the normal T9-Y0 dwarfs. We also note here that the J-band peak appears slightly narrower than that of the Y0 standard (see Figure 9). For this reason, we reclassify WISE $1405+5534$ as Y0.5. The nearinfrared colors of WISE $1405+5534$ support this change (see Section 5.3).

WISE 2209+2711 (Y0:-Cushing et al. 2014a), while noisy, shows a very peaked appearance, more similar to that of the Y1s than the Y0s. This may be providing a hint that this object is possibly $\mathrm{Y} 1$ or intermediate between $\mathrm{Y} 0$ and $\mathrm{Y} 1$.

\section{2. $\mathrm{NH}_{3}$}

The appearance of ammonia $\left(\mathrm{NH}_{3}\right)$ in the near-infrared spectra of cool brown dwarfs has been suggested for some time as the harbinger of the Y spectral class (Burrows et al. 2003; Leggett et al. 2007; Kirkpatrick 2008). Models predict that $\mathrm{NH}_{3}$ absorption bands could become prominent in brown dwarfs with temperatures $\lesssim 450 \mathrm{~K}$. Cushing et al. (2011) show a possible detection of $\mathrm{NH}_{3}$ on the blue wing of the $\mathrm{H}$-band peak of WISE $1738+2732$, but this $\mathrm{NH}_{3}$ feature is itself confused with a strong $\mathrm{H}_{2} \mathrm{O}$ band. An additional $\mathrm{NH}_{3}$ absorption component is predicted to emerge that will affect 

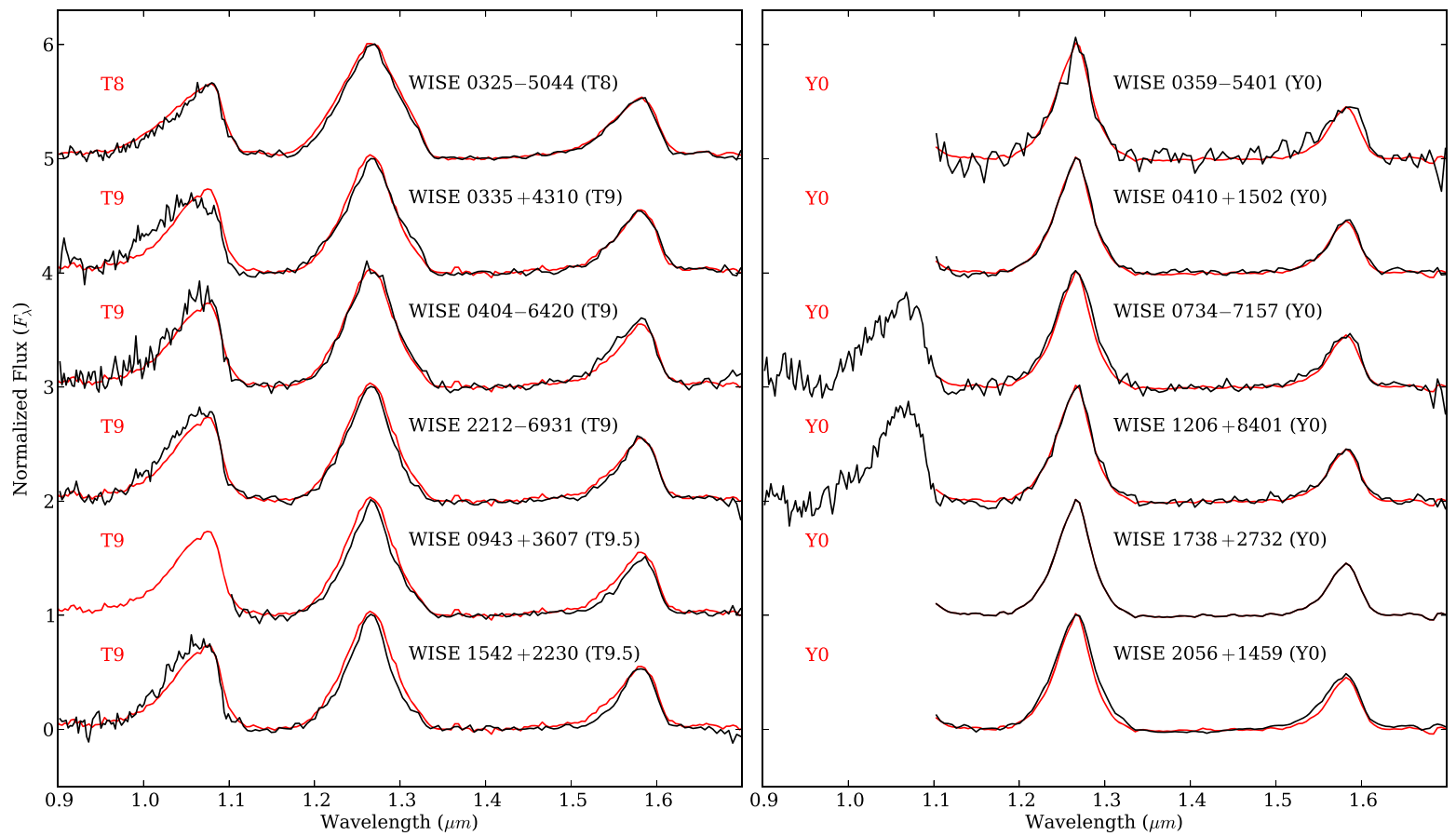

Figure 8. All HST spectra (black) ordered by spectral type, normalized at $1.27 \mu \mathrm{m}$, offset by constants, and compared with corresponding spectral standards (red). The spectral standards and the instruments from which the comparison spectra were obtained are as follows: T8-2MASSI J0415195-093506 (IRTF/SpeX; Burgasser et al. 2006), T9-UGPS J072227.51-054031.2 (IRTF/SpeX; Cushing et al. 2011), Y0-WISE J173835.53+273259.0 (HST/WFC3; Cushing et al. 2011), and Y1WISE J035000.32-565830.2 (HST/WFC3; Kirkpatrick et al. 2012).

The data used to create this figure are available.
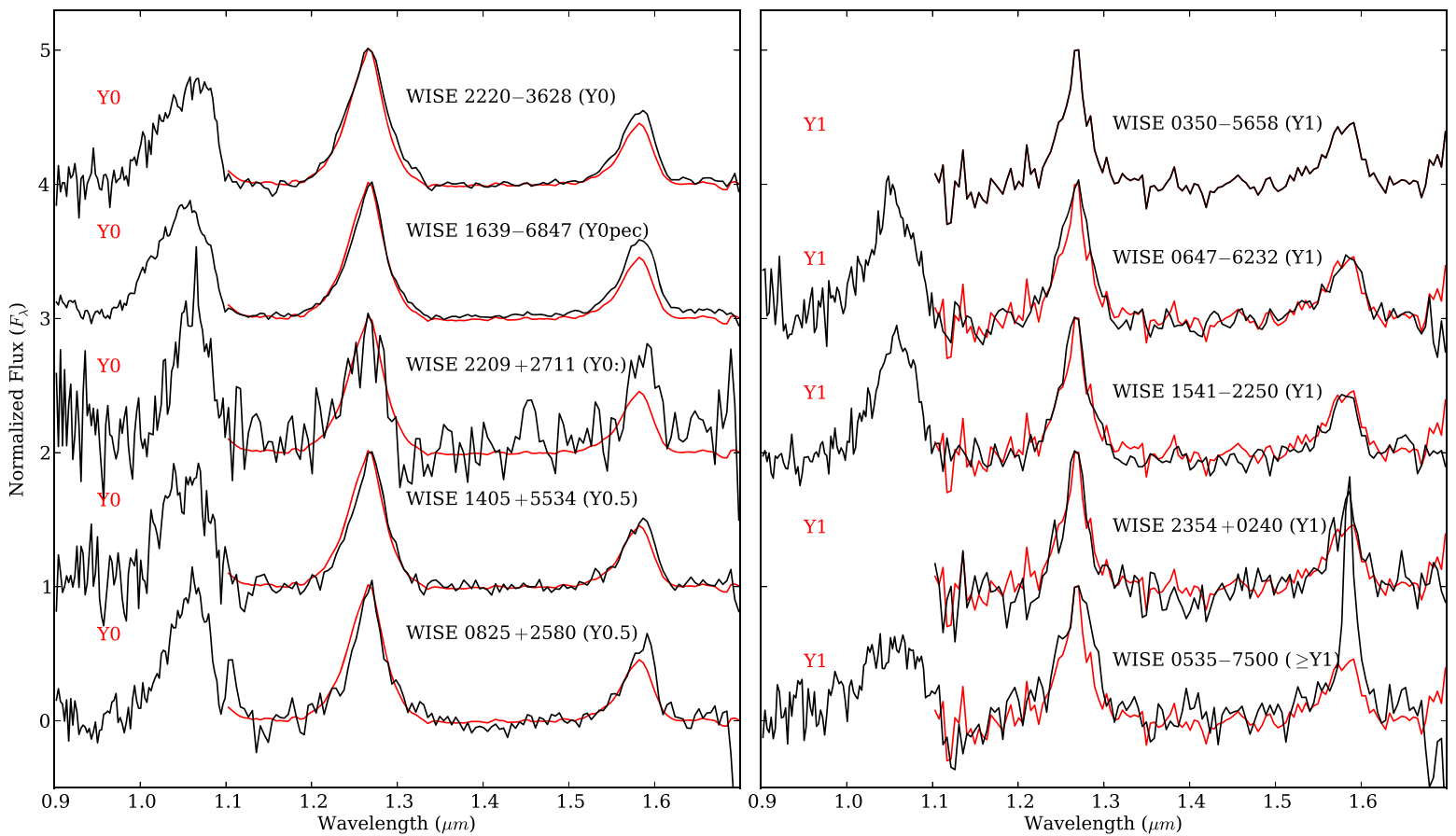

Figure 9. All HST spectra (black) ordered by spectral type, normalized at $1.27 \mu \mathrm{m}$, offset by constants, and compared with corresponding spectral standards (red). The spectral standards and the instruments from which the comparison spectra were obtained are as follows: T8-2MASSI J0415195-093506 (IRTF/SpeX; Burgasser et al. 2006), T9-UGPS J072227.51-054031.2 (IRTF/SpeX; Cushing et al. 2011), Y0-WISE J173835.53+273259.0 (HST/WFC3; Cushing et al. 2011), and Y1WISE J035000.32-565830.2 (HST/WFC3; Kirkpatrick et al. 2012).

The data used to create this figure are available.

the shape of the Y-band spectrum of extremely cool dwarfs, eventually causing a bifurcation of the peak around $300 \mathrm{~K}$. So far, a firm detection of $\mathrm{NH}_{3}$ absorption at $1.03 \mu \mathrm{m}$ has been elusive (Leggett et al. 2013). Leggett et al. (2013) have suggested that vertical mixing can explain the lack of $\mathrm{NH}_{3}$ in the spectrum of the Y0 dwarf WISE 2056+1459. Vertical 


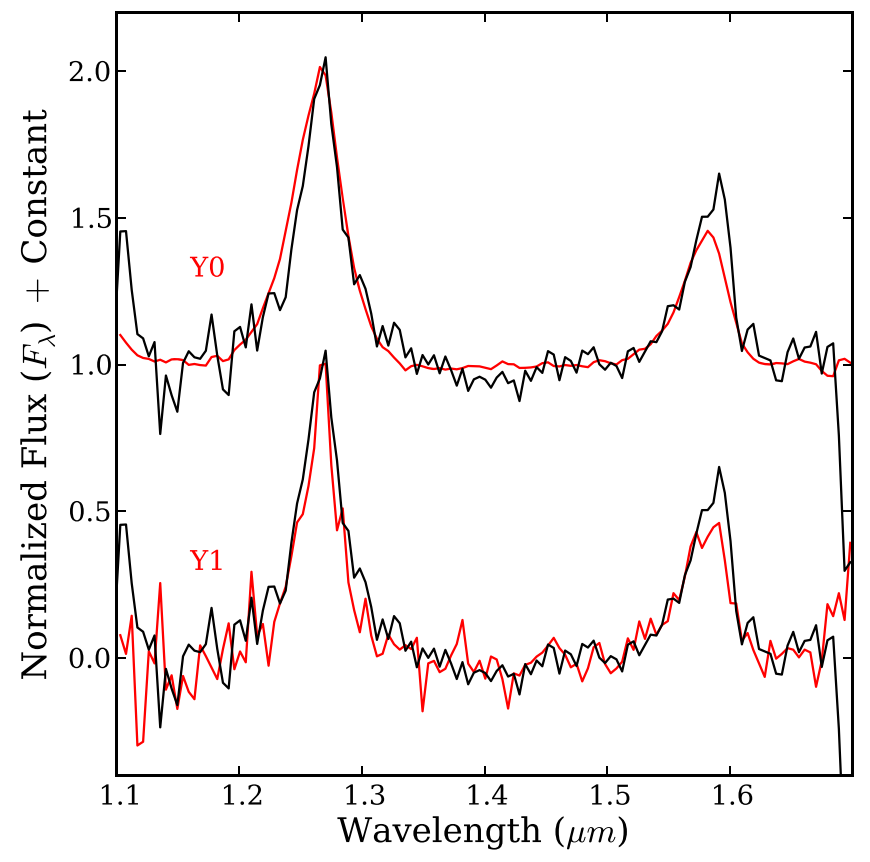

Figure 10. Spectral classification of WISE $0825+2805$. WISE $0825+2805$ is shown in black, while spectral standards are shown in red.

Table 4

Distance Estimates

\begin{tabular}{lccc}
\hline \hline AllWISE Name & $\begin{array}{c}\text { Spec. } \\
\text { Type }\end{array}$ & $\begin{array}{c}\text { Dist }^{\mathrm{a}} \\
(\mathrm{pc})\end{array}$ & $\begin{array}{c}\text { Dist }^{\mathrm{b}} \\
(\mathrm{pc})\end{array}$ \\
WISEA J032504.52-504403.0 & T8 & $36.4 \pm 2.4$ & $36.0 \pm 2.4$ \\
WISEA J040443.50-642030.0 & T9 & $24.8 \pm 0.7$ & $24.5 \pm 0.7$ \\
WISEA J082507.37+280548.2 & Y0.5 & $10.0 \pm 0.3$ & $10.9 \pm 0.3$ \\
WISEA J120604.25+840110.5 & Y0 & $14.5 \pm 0.4$ & $15.0 \pm 0.4$ \\
WISEA J221216.27-693121.6 & T9 & $16.8 \pm 0.5$ & $16.6 \pm 0.5$ \\
WISEA J235402.79+024014.1 & Y1 & $10.1 \pm 0.4$ & $11.9 \pm 0.5$ \\
\hline
\end{tabular}

Notes:

${ }^{a}$ Using W2 relation of Kirkpatrick et al. (2012).

${ }^{\mathrm{b}}$ Using W2 relation of Dupuy \& Liu (2012).

mixing keeps the atmospheric chemistry from reaching equilibrium, thus enhancing the $\mathrm{N}_{2}$ abundance and decreasing the $\mathrm{NH}_{3}$ abundance (Saumon et al. 2003; Hubeny \& Burrows 2007; Morley et al. 2014). Now, with an increased sample size of late-type brown dwarfs for which Y-band spectra are available, we can investigate the effects of $\mathrm{NH}_{3}$ in these atmospheres. If the lack of an $\mathrm{NH}_{3}$ detection in the spectrum of the Y0 dwarf WISE 2056+1459 is due to vertical mixing, as suggested by Leggett et al. (2013), then such a phenomenon may be ubiquitous in early $\mathrm{Y}$ dwarfs. We find no obvious evidence of the presence of $\mathrm{NH}_{3}$ in the $\mathrm{Y}$-band spectra for any of our late-type dwarfs.

\subsection{Colors}

We synthesized $Y-, J-$, and $H$-band photometry using the MKO-NIR photometric system for each brown dwarf in our sample. We also synthesized HST F105W, F125W, and F140W photometry using available system throughput tables. ${ }^{13}$ Uncertainties are computed using a Monte Carlo approach.

$\overline{13}$ http://stsci.edu/hst/wfc3/ins_performance/throughputs

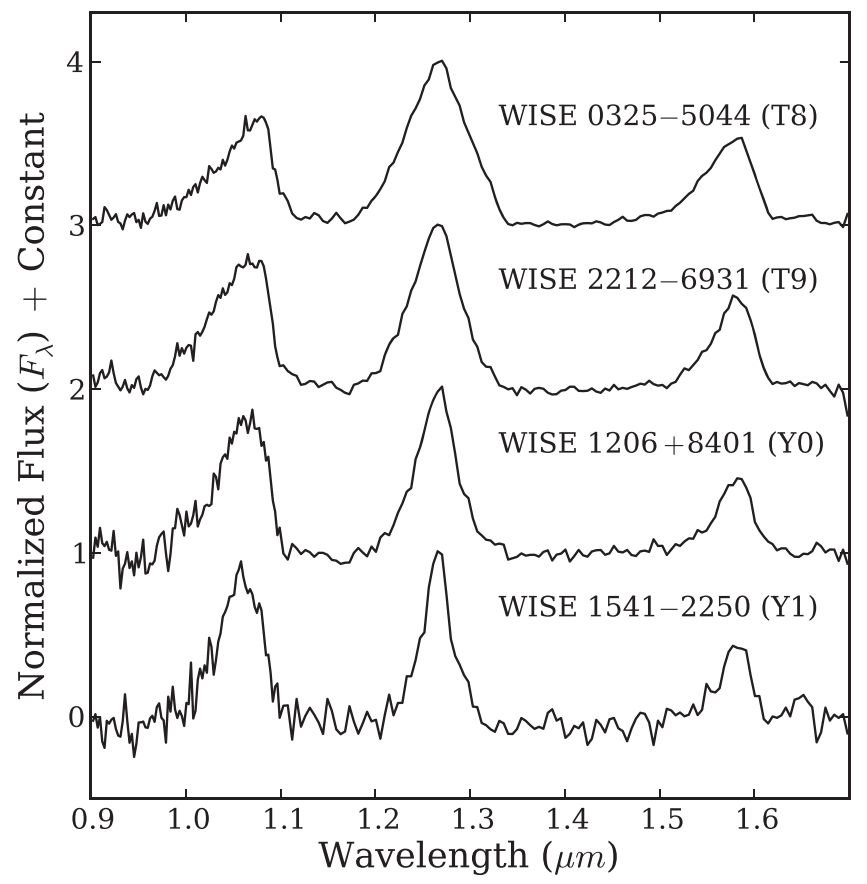

Figure 11. Complete spectral sequence from T8 to Y1.

For each spectrum, a value is selected from a normal distribution using the flux density at each wavelength as the mean and the flux density uncertainty as the standard deviation. Synthetic photometry is performed on the resulting spectrum, and the process is repeated 1000 times. We take the mean photometric value as the true value and the standard deviation of the distribution as the uncertainty. The resulting magnitudes and uncertainties are listed in Table 5. To investigate trends as a function of spectral type, we also synthesize photometry on the entire sample of $\mathrm{T}$ dwarfs available from the Spex Prism Spectral Library ${ }^{14}$ (as of June, 2014) using the same method described above. Colors as a function of spectral type are shown in Figure 13.

Our synthetic YJH photometry generally agrees well with published photometry from Cushing et al. (2011); Kirkpatrick et al. (2012); Morley et al. (2012); Dupuy \& Kraus (2013); Leggett et al. (2013); Beichman et al. (2014); Cushing et al. (2014a); Kirkpatrick et al. (2014), and Leggett et al. (2014), with only a few exceptions (56 out of 62 [87\%] measurements have differences that are within $3 \sigma$ of zero). Our measured $\mathrm{H}$ magnitude for WISE $0410+1502$ and $\mathrm{J}$ magnitude for WISE $1405+5534$ differ significantly from the measured values in Cushing et al. (2011) and Kirkpatrick et al. (2012), though they do agree with the values quoted in Leggett et al. (2013). Our J- and H-band magnitudes for WISE 1541-2250 disagree with the values given in Morley et al. (2012) and Leggett et al. (2014), respectively. Using our measured magnitudes, WISE 1541-2250 does not stand out prominently from the other Y1 dwarfs in color-color and color versus spectral type diagrams. Our measured J magnitude for WISE 1738+2732 disagrees with the value quoted in Leggett et al. (2013), though it does agree with the measurements from Cushing et al. (2011) and Kirkpatrick et al. (2012). Lastly, our measured J and $\mathrm{H}$ magnitudes for WISE 2056+1459 agree well with the measured values from Cushing et al. (2011) and Kirkpatrick et al.

\footnotetext{
${ }^{14}$ http://pono.ucsd.edu/ adam/browndwarfs/spexprism/
} 


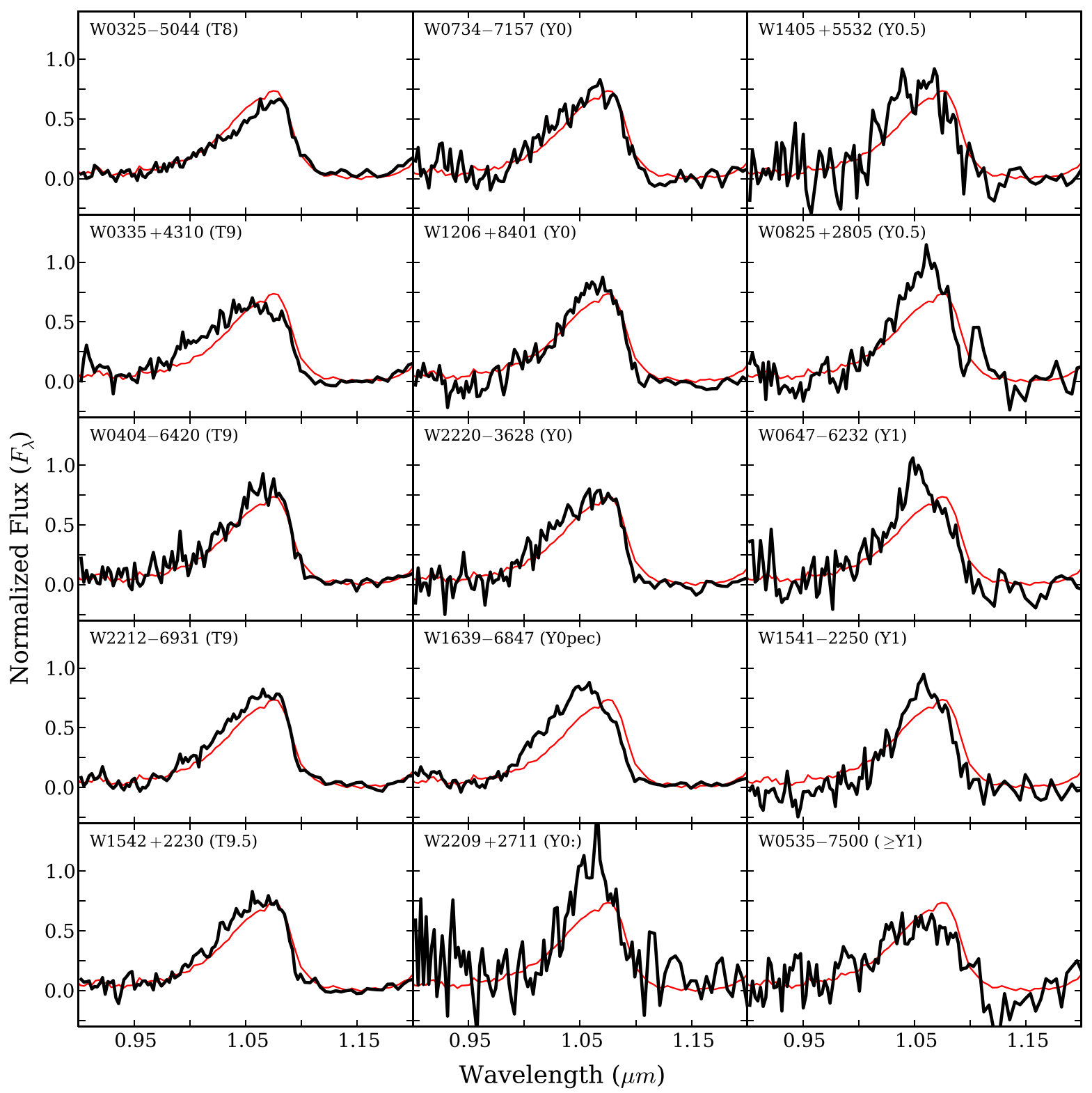

Figure 12. HST G102 spectra for WISE brown dwarfs (black). Each spectrum is normalized at $1.27 \mu \mathrm{m}$ (the J-band peak) and ordered sequentially by spectral type from top to bottom, then left to right. For comparison purposes, the spectrum of the T9 standard UGPS J072227.51-054031.2 is included in red on each plot.

(2012), though they do not agree with the values from Leggett et al. (2013). While we cannot rule out extreme variability as the source of magnitude differences, it is more likely that some measurements are erroneous. Since our photometry comes from our slitless grism spectroscopy, one potential source of error in our measurements is contamination from nearby bright objects or uneven background fluctuations, though our reduction process (Section 3.2) should minimize such effects.

Previous investigations of color/spectral type relations show distinct changes at the $\mathrm{T} / \mathrm{Y}$ boundary (Lodieu et al. 2013; Leggett et al. 2013, 2014). We see similar changes in Figure 13, namely, the $Y-J$ (and $F 105 W-F 125 W$ ) color plummets for spectral types later than T8, and the $J-H$ color plateaus at T7 and slowly turns redder for the Y dwarfs. As noted by Leggett et al. (2010), the blueward trend in the $Y-J$ colors of late-type dwarfs may be due to $\mathrm{K} \mathrm{I}$ condensing into $\mathrm{KCl}$ as temperatures decrease. This condensing weakens the broad $0.77 \mu \mathrm{m} \mathrm{K}$ I doublet, resulting in a brighter Y band. A similar trend is seen in the $z-J$ colors of Y dwarfs in Lodieu et al. (2013). The redward trend of the $J-H$ color for the latest Y dwarfs occurs because the J-band peak for these objects continues to narrow, while the H-band peak does not (see Figure 11).

Figure 14 shows the $Y-J$ (when available) versus $J-H$ and $J-H$ versus $J-W 2$ colors for our complete sample of brown dwarfs along with synthesized colors from the model spectra of Saumon et al. (2012); Morley et al. (2012), and Morley et al. (2014). While neither the cloudy nor cloud-free models reproduce the trends seen for our measured dwarfs, the cloudy models do a better job approximating the colors of our late-type dwarf sample than the cloud-free models. Morley et al. (2012) show that the inclusion of clouds is an efficient way to redden the $J-H$ colors of late-type brown dwarfs. Figure 14 also shows that models with lower surface gravities and lower $f_{\text {sed }}$ values do not fit the observed color trends as well. 
Table 5

Synthetic Photometry

\begin{tabular}{|c|c|c|c|c|c|c|c|}
\hline AllWISE Name & $\begin{array}{l}\text { Spec. } \\
\text { Type }\end{array}$ & $\begin{array}{l}\mathrm{Y}_{M K O} \\
(\mathrm{mag})\end{array}$ & $\begin{array}{c}\mathrm{J}_{M K O} \\
(\mathrm{mag})\end{array}$ & $\begin{array}{l}\mathrm{H}_{M K O} \\
(\mathrm{mag})\end{array}$ & $\begin{array}{c}\text { F105W } \\
(\mathrm{mag})\end{array}$ & $\begin{array}{c}\text { F125W } \\
(\mathrm{mag})\end{array}$ & $\begin{array}{c}\text { F140W } \\
(\mathrm{mag})\end{array}$ \\
\hline WISEA J032504.52-504403.0 & $\mathrm{T} 8$ & $19.980 \pm 0.027$ & $18.935 \pm 0.024$ & $19.423 \pm 0.027$ & $20.601 \pm 0.028$ & $19.547 \pm 0.023$ & $19.223 \pm 0.022$ \\
\hline WISEA J033515.07+431044.7 & T9 & $20.166 \pm 0.029$ & $19.467 \pm 0.023$ & $19.938 \pm 0.031$ & $20.939 \pm 0.033$ & $20.137 \pm 0.025$ & $19.785 \pm 0.023$ \\
\hline WISEA J035934.07-540154.8 ${ }^{\mathrm{a}}$ & Y0 & $\ldots$ & $21.566 \pm 0.046$ & $22.028 \pm 0.112$ & & $22.258 \pm 0.062$ & $21.789 \pm 0.045$ \\
\hline WISEA J040443.50-642030.0 & $\mathrm{T} 9$ & $20.328 \pm 0.032$ & $19.647 \pm 0.025$ & $19.970 \pm 0.033$ & $21.063 \pm 0.037$ & $20.293 \pm 0.028$ & $19.893 \pm 0.024$ \\
\hline WISEA J041022.7 & Y0 & $\ldots$ & $19.325 \pm 0.024$ & $19.897 \pm 0.038$ & $\ldots$ & $19.997 \pm 0.025$ & $19.643 \pm 0.024$ \\
\hline WISEA J073444.03-715743.8 & Y0 & $20.870 \pm 0.041$ & $20.354 \pm 0$. & $21.069 \pm 0$ & $21.675 \pm 0.051$ & $21.045 \pm 0$ & $20.726 \pm 0.030$ \\
\hline WISEA J082507.37+280548.2 & Y0.5 & $22.566 \pm 0.053$ & $22.401 \pm 0.050$ & $22.965 \pm 0.139$ & $23.409 \pm 0.073$ & $23.015 \pm 0.062$ & $22.731 \pm 0.051$ \\
\hline WISEA J094306.00+360723.3 & T9.5 & & $19.766 \pm 0.025$ & $20.315 \pm 0.038$ & & $20.444 \pm 0.027$ & $20.092 \pm 0.025$ \\
\hline WISEA J120604.25+840110.5 & Y0 & $20.875 \pm 0.036$ & $20.472 \pm 0.030$ & $21.061 \pm 0.062$ & $21.819 \pm 0.050$ & $21.171 \pm 0.036$ & $20.798 \pm 0.029$ \\
\hline WISEA J140518.32+553421.3 & Y0.5 & $21.333 \pm 0.057$ & $21.061 \pm 0.035$ & $21.501 \pm 0.073$ & $22.193 \pm 0.076$ & $21.730 \pm 0.044$ & $21.375 \pm 0.035$ \\
\hline WISE J154151.65-225024.9 & $\mathrm{Y} 1$ & $21.671 \pm 0.037$ & $21.631 \pm 0.064$ & $22.085 \pm 0.170$ & $22.724 \pm 0.083$ & $22.443 \pm 0.097$ & $22.138 \pm 0.078$ \\
\hline WISEA J221216.27-693121.6 & $\mathrm{T} 9$ & $20.282 \pm 0.023$ & $19.737 \pm 0.024$ & $20.225 \pm 0.036$ & $21.043 \pm 0.027$ & $20.378 \pm 0.026$ & $20.047 \pm 0.024$ \\
\hline WISEA J222055.34-362817.5 & Y0 & $20.899 \pm 0.034$ & $20.447 \pm 0.025$ & $20.858 \pm 0.035$ & $21.783 \pm 0.042$ & $21.131 \pm 0.027$ & $20.749 \pm 0.024$ \\
\hline WISEA J235402.79+024014. $1^{\mathrm{a}}$ & Y1 & $\ldots$ & $23.068 \pm 0.199$ & $22.882 \pm 0.300$ & $\ldots$ & $24.124 \pm 0.393$ & $23.292 \pm 0.179$ \\
\hline
\end{tabular}

Notes:

${ }^{\text {a }}$ Y-band and F105W photometry were not synthesized for these dwarfs because they were not observed with the G102 grism.

${ }^{\mathrm{b}}$ H-band and F140W photometry were not synthesized for WISE $0535-7500$ because its spectrum is contaminated in this wavelength range.
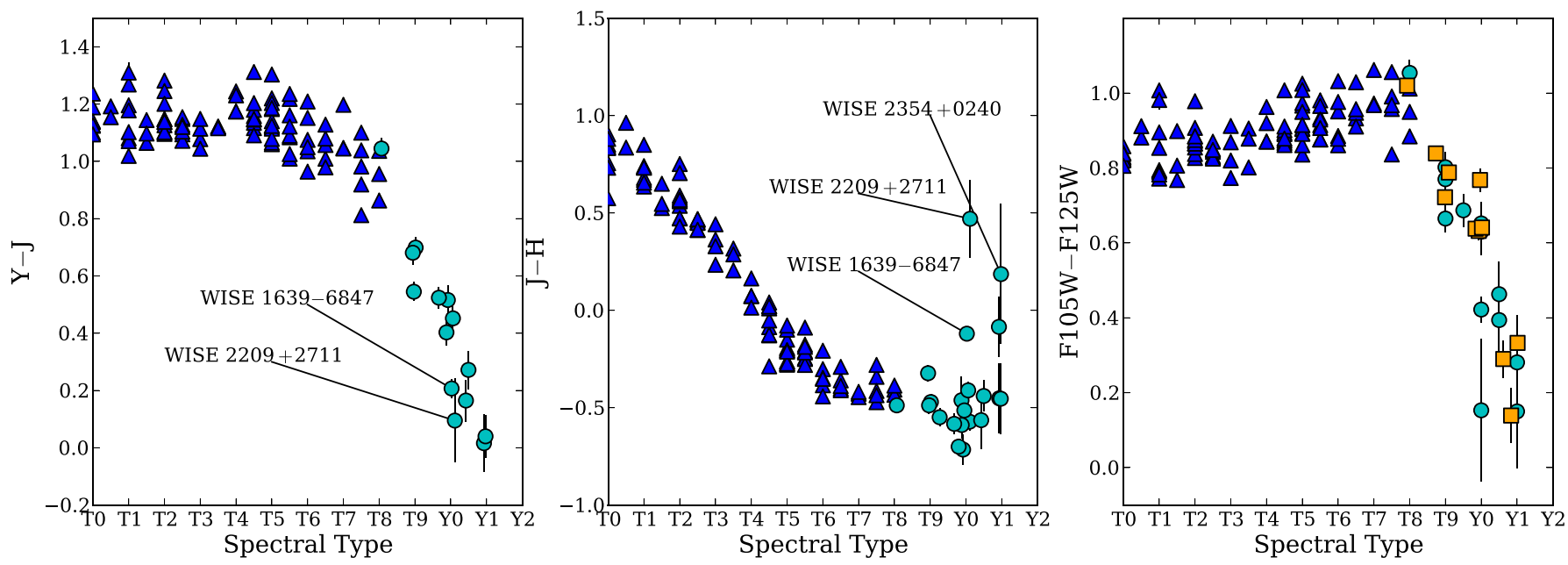

Figure 13. Left: $Y-J$ color as a function of spectral type for $\mathrm{T}$ and $\mathrm{Y}$ dwarfs. $\mathrm{T}$ dwarfs from the SpeX Prism Spectral Library are represented by blue triangles, while synthesized colors of brown dwarfs in this study are represented by cyan circles. Small offsets have been added along the abscissa for differentiation purposes. Center: $J-H$ color as a function of spectral type for T and Y dwarfs. Right: HST colors as a function of spectral type for T and Y dwarfs. Synthetic photometry of brown dwarfs in this study are represented by cyan circles, while colors found using aperture photometry are represented by orange squares.

\subsection{Physical Parameters}

The effective temperatures of brown dwarfs are typically estimated in two ways. If the distance to a brown dwarf is known and a significant fraction of its emergent flux can be measured, then its bolometric luminosity can be computed. Since brown dwarfs all have similar radii due to the equation of state of their partial degenerate interiors, an effective temperature can then be computed using the Stefan Boltzmann law (e.g., Golimowski et al. 2004; Vrba et al. 2004; Dupuy \& Kraus 2013). Alternatively, model spectra can be compared directly to observed spectra and/or photometry to infer not only the effective temperatures of brown dwarfs but also their surface gravity and metallicity (e.g., Cushing et al. 2008; Witte et al. 2011; Leggett et al. 2013). Because accurate distances to many of the dwarfs in our sample are not known, we have taken the second approach and compared our near-infrared spectra and mid-infrared photometry to atmospheric models in order to estimate their atmospheric parameters.

In particular, we compare are spectra and photometry to several different sets of solar-metallicity models, including the cloud-free models from Saumon et al. (2012), the cloudy models of Morley et al. (2012), and the cloudy models of 

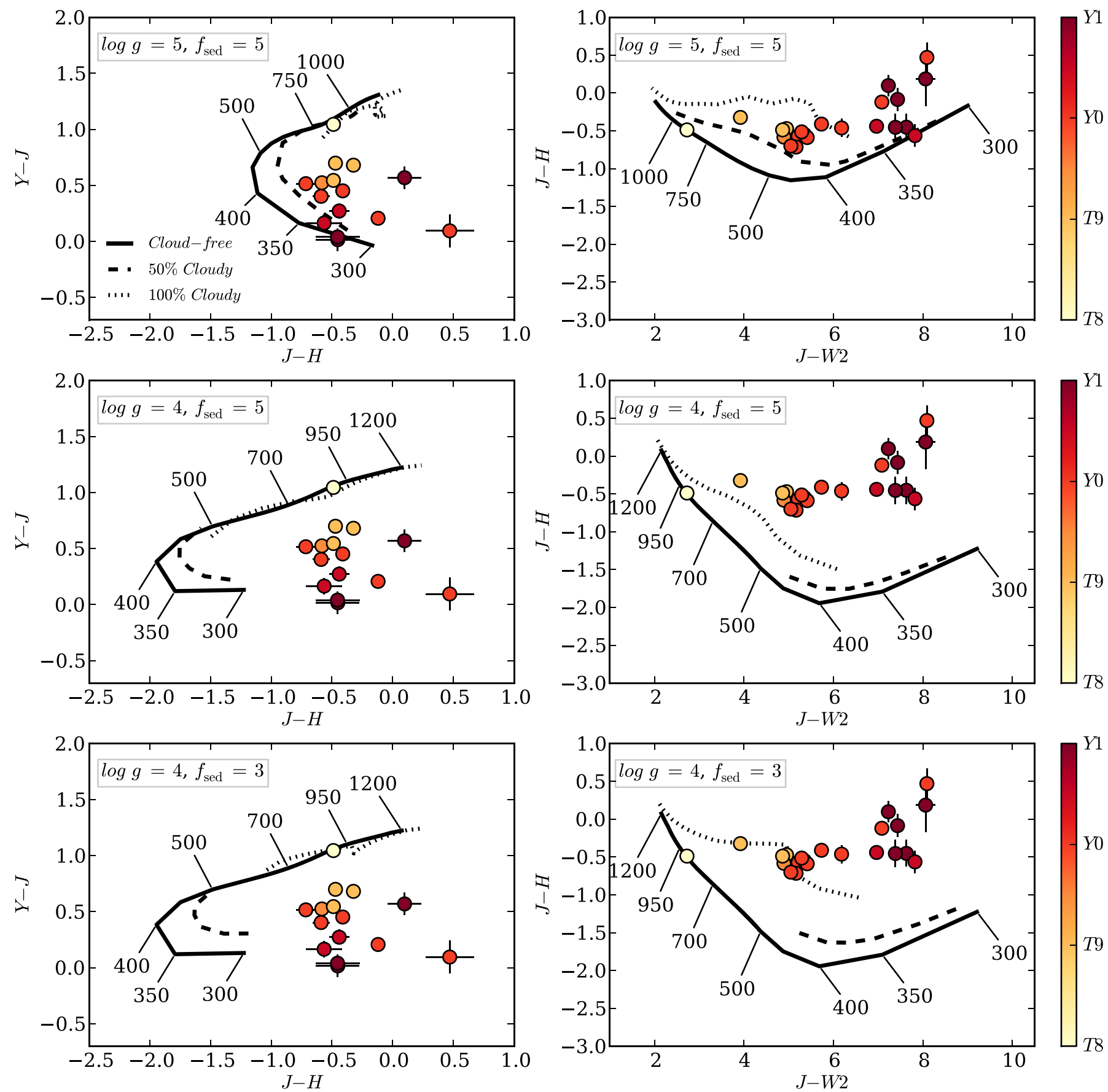

Figure 14. Left: $J-H$ versus $Y-J$ synthesized colors for brown dwarfs from our sample, cloud-free Saumon et al. (2012) models, and cloudy models from Morley et al. (2012) and Morley et al. (2014). Right: $J-W 2$ vs. $J-H$ for brown dwarfs from our sample with the Saumon et al. (2012); Morley et al. (2012), and Morley et al. (2014) models. $f_{\text {sed }}$ values apply to the cloudy and partly cloudy models (not the cloud-free models) in all panels.

Morley et al. (2014). The latter set is a new suite of models that, in addition to including sulfide and chloride condensates, also includes water-ice clouds for objects with $\mathrm{T}_{\text {eff }} \leqslant 450 \mathrm{~K}$. Some models from Morley et al. (2014) are also "partly cloudy", calculated with 50\% cloudy and 50\% hole surface coverage. The models are arranged in a grid of $\mathrm{T}_{\text {eff }}, \log g$, and $f_{\text {sed }}$ values. The $f_{\text {sed }}$ parameter describes the efficiency of cloud sedimentation in the models, where larger $f_{\text {sed }}$ values correspond to larger grain sizes, which lead to an increased sedimentation efficiency and hence thinner clouds. We adopt the $f_{\text {sed }}=$ "nc" or "no clouds" notation of Cushing et al. (2008) to refer to the cloud-free models. Table 6 summarizes the $\mathrm{T}_{\text {eff }}$, $\log g$, and $f_{\text {sed }}$ combinations that were used for model fitting.

In order to compare the models to our observed spectra, each model spectrum was resampled to be uniform in $\ln (\lambda)$ space, smoothed with a Gaussian kernel to a resolving power of $H S T$ WFC3, and resampled onto the same wavelength grid as the HST WFC3 spectrum. Synthetic flux density values in the [3.6] and [4.5] Spitzer bands were computed from the models as described in Cushing et al. (2006). Following Cushing et al. 
Table 6

Models Used for Spectral Fitting ${ }^{\mathrm{a}}$

\begin{tabular}{|c|c|c|c|c|}
\hline $\begin{array}{l}\mathrm{T}_{\text {eff }} \\
(\mathrm{K})\end{array}$ & $\begin{array}{c}\log g \\
\left(\mathrm{~cm} \mathrm{~s}^{-1}\right)\end{array}$ & $f_{\text {sed }}$ & $\begin{array}{l}\text { Cloud } \\
\text { Type } \\
(\%)\end{array}$ & Reference \\
\hline $400-700(50), 700-1000(100)$ & 3.0 & $\mathrm{nc}$ & $\ldots$ & 1 \\
\hline $400-1000(50)$ & 3.5 & $\mathrm{nc}$ & $\ldots$ & 1 \\
\hline $300-1000(50)$ & 3.75 & $\mathrm{nc}$ & $\ldots$ & 1 \\
\hline $300-1000(50)$ & 4.0 & $\mathrm{nc}$ & $\ldots$ & 1 \\
\hline $400-1000(50)$ & 4.25 & $\mathrm{nc}$ & $\ldots$ & 1 \\
\hline $300-1000(50)$ & 4.5 & $\mathrm{nc}$ & $\cdots$ & 1 \\
\hline $400-1000(50)$ & 4.75 & $\mathrm{nc}$ & $\cdots$ & 1 \\
\hline $400-1000(50)$ & 5.0 & $\mathrm{nc}$ & $\ldots$ & 1 \\
\hline $400-1000(100)$ & 5.5 & $\mathrm{nc}$ & $\ldots$ & 1 \\
\hline $200-400(25)$ & 3.0 & 5 & $50^{\mathrm{b}}$ & 2 \\
\hline $200-400(25), 450$ & 3.5 & 5 & $50^{\mathrm{b}}$ & 2 \\
\hline $200-400(25), 450$ & 4.0 & 3 & $50^{\mathrm{b}}$ & 2 \\
\hline $200-400(25), 450$ & 4.0 & 5 & $50^{\mathrm{b}}$ & 2 \\
\hline $200-400(25), 450$ & 4.0 & 7 & $50^{\mathrm{b}}$ & 2 \\
\hline $200-400(25), 450$ & 4.5 & 5 & $50^{\mathrm{b}}$ & 2 \\
\hline $\begin{array}{l}200-400(25), 400-700(50), \\
\quad 700-1000(100)\end{array}$ & 5.0 & 5 & $50^{\mathrm{b}}$ & 2 \\
\hline $400,450,550,600-1000(100)$ & 4.0 & 2 & 100 & 3 \\
\hline $400-600(50), 600-1000(100)$ & 4.0 & 3 & 100 & 3 \\
\hline $400-600(50), 600-1000(100)$ & 4.0 & 4 & 100 & 3 \\
\hline $400-600(50), 600-1000(100)$ & 4.0 & 5 & 100 & 3 \\
\hline $400-600(50), 600-1000(100)$ & 4.5 & 2 & 100 & 3 \\
\hline $400-600(50), 600-1000(100)$ & 4.5 & 3 & 100 & 3 \\
\hline $400-600(50), 600-1000(100)$ & 4.5 & 4 & 100 & 3 \\
\hline $400-600(50), 600-1000(100)$ & 4.5 & 5 & 100 & 3 \\
\hline $\begin{array}{l}460,500-600(50) \\
\quad 600-1000(100)\end{array}$ & 5.0 & 2 & 100 & 3 \\
\hline $\begin{array}{l}400-600(50), 460 \\
600-1000(100)\end{array}$ & 5.0 & 3 & 100 & 3 \\
\hline $\begin{array}{l}400,460,500-600(50), \\
\quad 600-1000(100)\end{array}$ & 5.0 & 4 & 100 & 3 \\
\hline $\begin{array}{l}400,460,500-600(50) \\
\quad 600-1000(100)\end{array}$ & 5.0 & 5 & 100 & 3 \\
\hline $\begin{array}{l}400,500-600(50) \\
600-1000(100)\end{array}$ & 5.5 & 3 & 100 & 3 \\
\hline
\end{tabular}

Notes:

References: (1) Saumon et al. 2012; (2) Morley et al. 2014; (3) Morley et al. 2012

${ }^{\mathrm{a}}$ When a range is given, the value given in parentheses is the spacing for that interval.

${ }^{\mathrm{b}}$ With water clouds.

(2008), we evaluate a goodness-of-fit parameter for each model spectrum, defined as

$$
G_{k}=\sum_{i=1}^{n}\left(\frac{f_{i}-C_{k} \mathcal{F}_{k, i}}{\sigma_{i}}\right)^{2},
$$

where $n$ corresponds to each data pixel, $f_{i}$ is the flux density of the data, $\mathcal{F}_{k, i}$ is the flux density of the model $k$, and $\sigma_{i}$ are the errors for each observed flux density. The $C_{k}$ parameter is a multiplicative constant given by

$$
C_{k}=\frac{\sum f_{i} \mathcal{F}_{k, i} / \sigma_{i}^{2}}{\sum \mathcal{F}_{k, i} / \sigma_{i}^{2}} .
$$

$C_{k}$ is equivalent to $\left(R^{2} / d^{2}\right)$, where $R$ is the stellar radius and $d$ is the distance. For each model we compute a $C_{k}$ value and corresponding $G_{k}$ value and identify the best-fitting model as that model with the lowest overall $G_{k}$ value. Because in most instances the difference between the best-fitting models for each dwarf is marginal, we proved a range of best-fit model parameters from the three best-fitting models for each brown dwarf in Table 7 . The $\left(R^{2} / d^{2}\right)$ and $\chi^{2}$ values in Table 7 refer to the model with the best fit.

The derived effective temperatures are generally correlated with spectral type in that the effective temperatures decrease with increasing spectral type: WISE 0325-5044 (the lone T8) has an effective temperature range of $550-600 \mathrm{~K}$, the T9 dwarfs have temperatures between 500 and $600 \mathrm{~K}$, the T9.5 dwarfs have temperatures between 450 and $500 \mathrm{~K}$, the Y0 dwarfs have temperatures between 400 and $450 \mathrm{~K}$, the Y1 dwarfs have temperatures between 300 and $400 \mathrm{~K}$. Exceptions include the Y0: dwarf WISE 2209+2711 $\left(\mathrm{T}_{\text {eff }}=500-550 \mathrm{~K}\right)$ and the Y1 dwarf WISE 0535-7500, which is located in an extremely crowded field and thus has contamination issues (see Section 5.1).

A comparison with the effective temperatures derived from bolometric luminosities in Dupuy \& Kraus (2013) finds some agreement, though there are several discrepancies. The main discrepancies appear for the objects with very uncertain parallaxes in Dupuy \& Kraus (2013) (WISE 0359-5401, WISE 0535-7500, WISE 1541-2250, and WISE 1639-6847). For these objects with uncertain parallaxes, two (WISE 0359-5401 and WISE 1639-6847) are found to have $\mathrm{T}_{\text {eff }}$ values much colder in Dupuy \& Kraus (2013) than our modelfitting derived values, while the other two (WISE 0535-7500 and WISE 1541-2250) are found to be much warmer. It is unclear whether these discrepancies are solely due to parallax uncertainties or other systematic errors (see below).

Although atmospheric models can be computed for any given $\mathrm{T}_{\text {eff }} / \log g$ values, not all combinations are physical because theoretical evolutionary models limit the $\mathrm{T}_{\text {eff }} / \log g$ values that brown dwarfs can obtain. We therefore compared our derived $\mathrm{T}_{\text {eff }} / \log g$ values to the low-mass, solar-metallicity, cloud-free evolutionary models of Saumon \& Marley (2008) in order to confirm that our best-fit models are all physical (Figure 15). We note that the atmospheric models used in our analysis were not used as boundary conditions for the evolutionary models and thus our comparisons are not technically self-consistent. Inspection of Figure 15 suggests that several of the derived surface gravities are not physical (those objects with $\log g$ values of 5 and $T_{\text {eff }}$ estimates $\leqslant 400$ $\mathrm{K})$. Evolutionary models can also be used to estimate the ages of brown dwarfs. Most of our sources have ages of several Gyr, as expected for objects near to the Sun, though some objects were found to have younger (a few hundred Myr) or older $(>5$ Gyr) ages than expected. Indeed, a trend is apparent whereby the earlier spectral types in our sample (T8 and T9) are preferentially fit with lower $\log g$ values and hence younger ages, while the latest spectral types (Y0.5 and Y1) are preferentially fit with larger log $g$ values (some of which are unphysical). Although it is possible that this trend is real, it is more likely a result of systematic errors in the model fits. Fits with unphysical gravities are indicated as such in the age column of Table 7.

While the derived temperatures are generally consistent with previous work, the fits to the data are, in many cases, rather poor. The discrepancies between the data and models generally fall into two categories. Because the HST spectra contain over 
Table 7

Model-derived Physical Characteristics ${ }^{\mathrm{a}}$

\begin{tabular}{|c|c|c|c|c|c|c|c|c|}
\hline AllWISE Name & $\begin{array}{l}\text { Spec. } \\
\text { Type }\end{array}$ & $\begin{array}{l}\mathrm{T}_{\text {eff }} \\
(\mathrm{K})\end{array}$ & $\begin{array}{c}\log g \\
\left(\mathrm{~cm} \mathrm{~s}^{-1}\right)\end{array}$ & $f_{\text {sed }}$ & $\begin{array}{c}\text { \% Cloudy } \\
(\%)\end{array}$ & $\begin{array}{c}(\mathrm{R} / \mathrm{d}) \\
\left(\mathrm{R}_{\text {jup }} / \mathrm{pc}\right)\end{array}$ & $\chi^{2} /$ d.o.f & $\begin{array}{l}\mathrm{Age}^{\mathrm{b}} \\
(\mathrm{Gyr})\end{array}$ \\
\hline WISEA J032504.52-504403.0 & $\mathrm{T} 8$ & $550-600$ & 4.0 & $3-4$ & 100 & $2.468 \times 10^{-2}$ & $5213 / 211$ & $0.08-0.3$ \\
\hline WISEA J033515.07+431044.7 & T9 & $500-550$ & $4.0-4.5$ & $3-5$ & 100 & $1.973 \times 10^{-2}$ & $5276 / 211$ & $0.2-1.5$ \\
\hline WISEA J035934.07-540154.8 & Y0 & 400 & $4.0-5.0$ & $2,3,5$ & 100 & $3.641 \times 10^{-2}$ & $2494 / 128$ & $1.5-8$ \\
\hline WISEA J040443.50-642030.0 & T9 & $550-600$ & 4.0 & $3-4$ & 100 & $1.861 \times 10^{-2}$ & $4458 / 211$ & $0.08-0.3$ \\
\hline WISEA J041022.75+150247.9 & Y0 & 400 & $4.0-4.5$ & $2,3,4$ & 100 & $1.007 \times 10^{-1}$ & $5172 / 128$ & $1.5-8$ \\
\hline WISEA J073444.03-715743.8 & Y0 & 450 & $4.0-4.5$ & $3-5$ & 100 & $2.410 \times 10^{-2}$ & $3109 / 211$ & $0.4-2$ \\
\hline WISEA J082507.37+280548.2 & Y0.5 & 400 & $4.5-5.0$ & $\ldots, 4,5$ & 0,100 & $1.808 \times 10^{-2}$ & $3313 / 211$ & $3-U$ \\
\hline WISEA J094306.00+360723.3 & T9.5 & $450-500$ & $4.0-4.5$ & $2,3,5$ & 100 & $4.167 \times 10^{-2}$ & $4506 / 128$ & $0.3-2$ \\
\hline WISEA J120604.25+840110.5 & Y0 & $400-450$ & $4.0-4.5$ & 3,5 & 100 & $4.040 \times 10^{-2}$ & $3475 / 211$ & $0.4-3$ \\
\hline WISEA J140518.32+553421.3 & Y0.5 & $350-400$ & $5.0-5.5$ & $\ldots, 5$ & 0,100 & $3.218 \times 10^{-2}$ & $4117 / 211$ & $\mathrm{U}$ \\
\hline WISE J154151.65-225024.9 & $\mathrm{Y} 1$ & 400 & $4.0-4.5$ & $\ldots$ & 0 & $2.072 \times 10^{-2}$ & $4102 / 211$ & $0.6-3$ \\
\hline WISEA J220905.75+271143.6 & Y0: & $500-550$ & $4.0-4.5$ & 4,5 & 100 & $6.682 \times 10^{-3}$ & $3106 / 211$ & $0.2-1.5$ \\
\hline WISEA J221216.27-693121.6 & T9 & $500-600$ & 4.0 & 3,5 & 100 & $1.541 \times 10^{-2}$ & $5389 / 211$ & $0.08-0.4$ \\
\hline WISEA J222055.34-362817.5 & Y0 & $400-450$ & $4.0-5.0$ & $2,3,5$ & 100 & $3.204 \times 10^{-2}$ & $4574 / 211$ & $1-6$ \\
\hline WISEA J235402.79+024014.1 & Y1 & $300-400$ & $4.0-5.0$ & 2,5 & 50,100 & $5.961 \times 10^{-2}$ & $10663 / 128$ & $1.5-\mathrm{U}$ \\
\hline
\end{tabular}

Notes:

${ }^{\mathrm{a}}$ Model-derived parameters are generally unreliable for reasons explained in Section 5.4 .

${ }^{\mathrm{b}}$ Ages are derived for the $\mathrm{T}_{\text {eff }}$ and $\log g$ ranges in columns 3 and 4 using the Saumon \& Marley (2008) cloudless evolutionary models. Some combinations of $\mathrm{T}_{\text {eff }}$ and $\log g$ derived from the atmospheric fits are unphysical in that brown dwarfs cannot evolve to have such values, so the corresponding ages are denoted as "U" for unphysical.

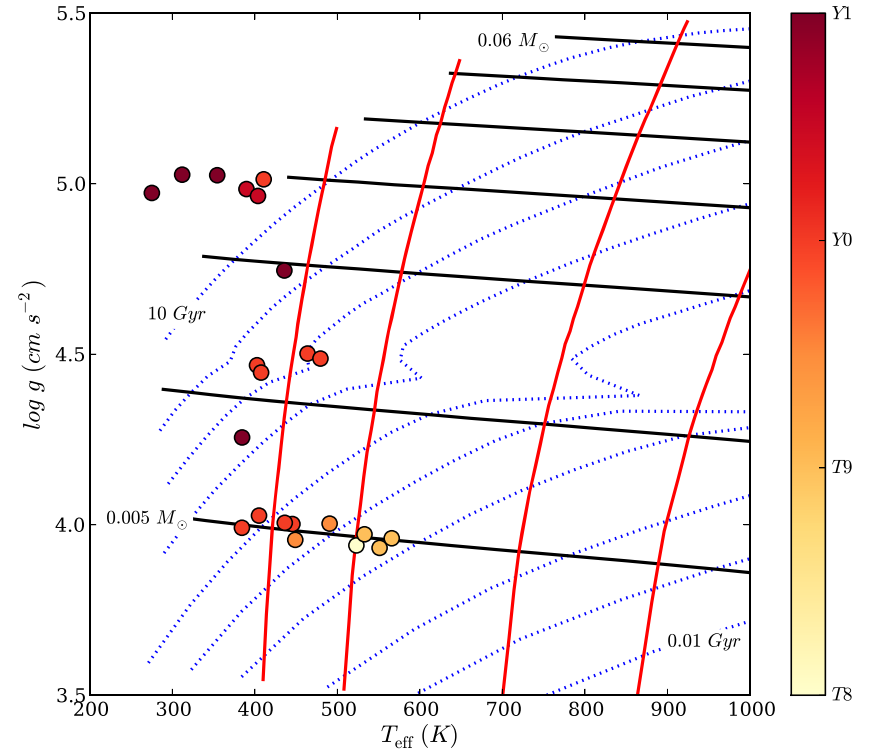

Figure 15. Best-fitting model parameters from Table 7 compared with the cloud-free evolutionary models from Saumon \& Marley (2008). Lines of constant mass are plotted in solid black for 0.005, 0.01, 0.02, 0.03, 0.04, 0.05, and $0.06 M_{\odot}$ from bottom to top. Lines of constant luminosity are plotted in solid red for $-6.4,-6.0,-5.4$, and $-5.0 \log \left(L / L_{\odot}\right)$ from left to right. Isochrones are plotted as dotted blue lines for 10, 4, 2, 1, 0.4, 0.2, 0.1, 0.04, 0.02, and 0.01 Gyr from left to right. Small offsets have been added along the abscissa and ordinate for differentiation purposes.

200 data points, the two IRAC points carry little weight in the fitting process, and thus the models "match" the near-infrared data reasonably well but often miss the Spitzer/IRAC points badly. Figure 16 shows the best fits to the T8 to Y1 spectral sequence from Figure 12. In some instances (e.g., WISE 0325-5044, WISE 1541-2250), the Spitzer model fluxes can be off by factors of 0.2 to 4 , respectively. Since much of the energy of cold brown dwarfs emerges at $5 \mu \mathrm{m}$, the bolometric luminosity of the best-fitting models disagrees with the observations by similar factors. A factor of 2 to 5 in $L_{b o l}$ can result in an effective temperature difference of up to $200 \mathrm{~K}$ (see Figure 15).

Closer inspection also reveals large differences between the models and observations in the shapes of the near-infrared bands. In particular, the heights of the model $H$-band peaks are significantly different from the observations, with the observed H-band peak consistently being higher than that of the bestfitting model. The height, width, and position of the J-band peak are generally well matched to the models. For the two latest spectral types, the J-band peak of the observations does not reach the same heights as those from the best-fitting models. The Y-band peaks for the two $\mathrm{T}$ dwarfs match well with the models, while the fits to the Y-band peaks of the Y dwarfs do not. The main discrepancy in the Y-band spectra of the $\mathrm{Y}$ dwarfs is the absence in the observations of the $\mathrm{NH}_{3}$ absorption feature expected to occur for temperatures $<500 \mathrm{~K}$. The problems with these particular model fits are nicely summarized by the color-color diagrams shown in Figure 14 .

Some of the issues in the near-infrared are likely a result of the fact that the models do not account for non-equilibrium $\mathrm{NH}_{3}$ chemistry due to vertical mixing in the atmosphere (see Section 5.2). Figure 17 shows the near-infrared spectrum of WISE 1541-2250 (Y1) along with the cross-section spectrum of $\mathrm{NH}_{3}$. Ammonia absorption shapes the entire near-infrared 


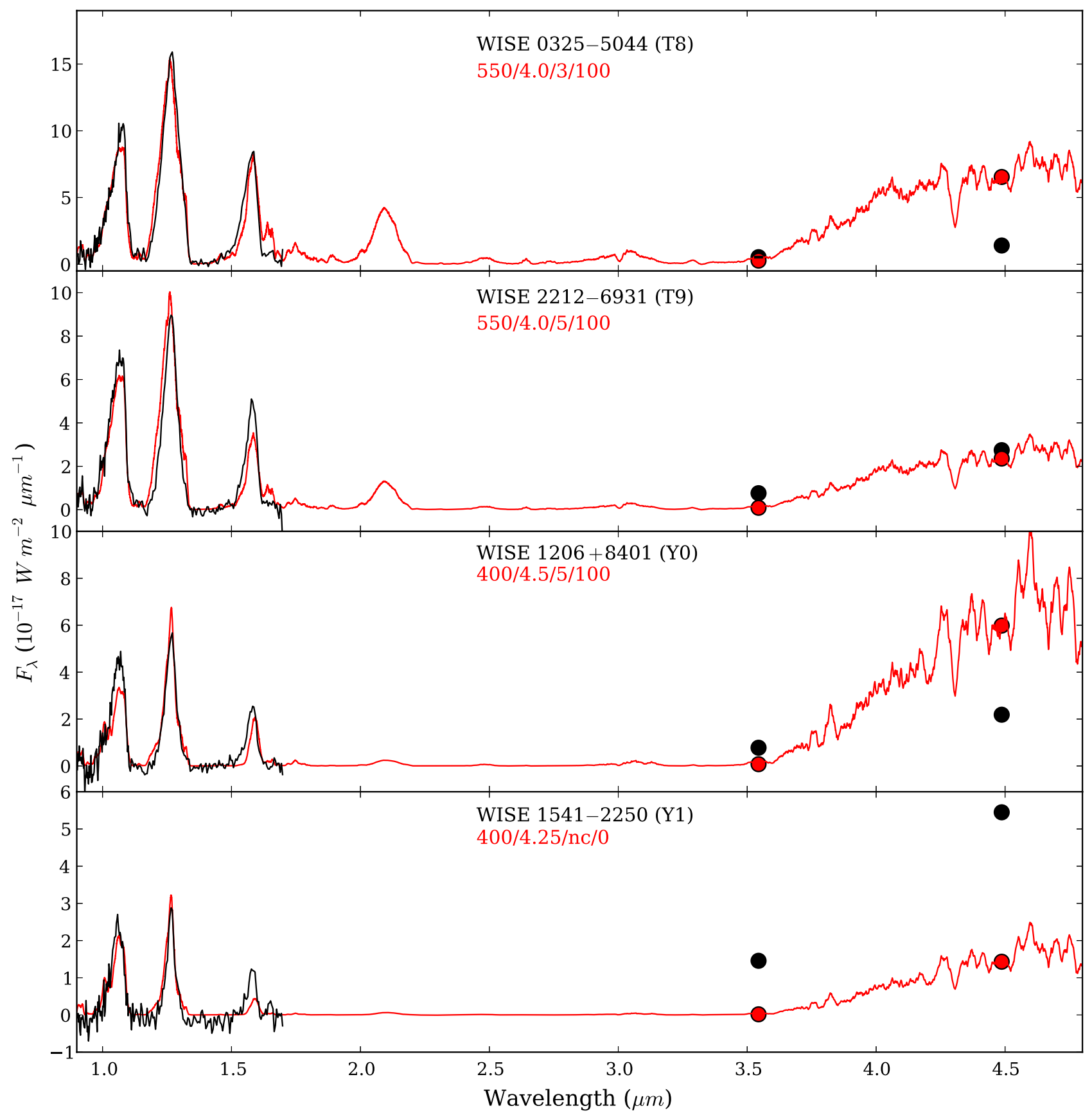

Figure 16. $\mathrm{T} 8$ to $\mathrm{Y} 1$ spectral sequence (black) with best-fit models (red). The best-fitting model parameters $\left(\mathrm{T}_{\text {eff }}\right.$ in $\mathrm{K}, \log g$ in $\mathrm{cm} \mathrm{s}^{-1}, f_{\text {sed }}$, and the percentage of cloud cover) are given under each object name. The black symbols represent the Spitzer IRAC photometry from Table 2, while the red circles indicate the synthetic photometry of the model in the chl and ch2 bandpasses. Error bars for the IRAC photometry are smaller than the symbol size.

spectrum-from the blue wing of the $H$-band peak, to the width of the $J$-band peak, to the shape of the $Y$-band peak. Morley et al. (2014) show that when all else is equal, the decrease in the $\mathrm{NH}_{3}$ abundance broadens the $J$ - and $H$-band peaks and removes the strong $\mathrm{NH}_{3}$ absorption feature in the $Y$ band. The inclusion of non-equilibrium chemistry, which would result in significant changes to the model spectra, would no doubt result in different effective temperature and surface gravity estimates. Based on the $H$ band alone, the change would be $300 \mathrm{~K}$ at a fixed gravity. Given the significant mismatches identified between these model spectra and data, we conclude that we cannot reliably derive reliable atmospheric parameters by fitting these model spectra to the observed spectra and photometry of our sample.

Leggett et al. (2014) state that the $Y, H$, and chl model fluxes are too low by about a factor of two compared to photometric measurements for late-type brown dwarfs. By extension, this suggests that the $J$ and $W 2$ magnitudes are not affected and thus the $J-W 2$ color may be able to provide a reasonable effective temperature estimate when compared with colors from model spectra. Figure 18 shows the $J-W 2$ color as a function of effective temperature for three different models - a cloud-free model from Saumon et al. (2012) $(\log g=5)$, a $50 \%$ cloud coverage model from Morley et al. (2014) (log 


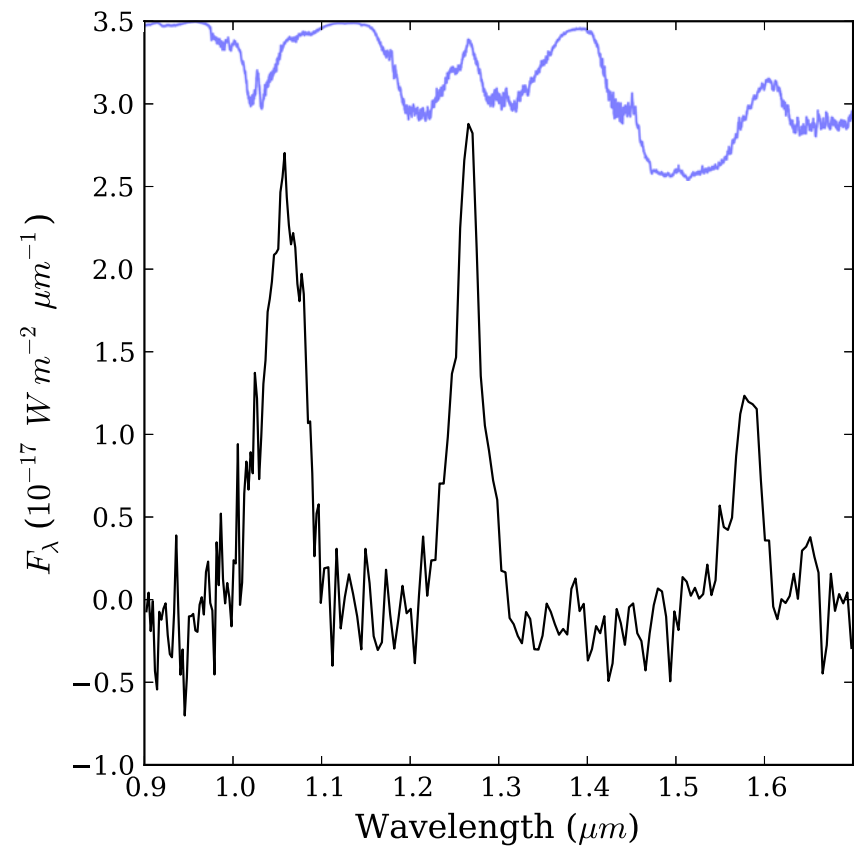

Figure 17. The near-infrared spectrum of WISE 1541-2250. The cross-section spectrum of $\mathrm{NH}_{3}$ at $\mathrm{T}=600 \mathrm{~K}$ and $\mathrm{P}=1$ bar (Richard Freedman, private communication), indicating the location of prominent $\mathrm{NH}_{3}$ absorption bands, is shown in blue. The $\mathrm{NH}_{3}$ cross-section spectrum is normalized at $1.033 \mu \mathrm{m}$, inverted, and offset for clarity.

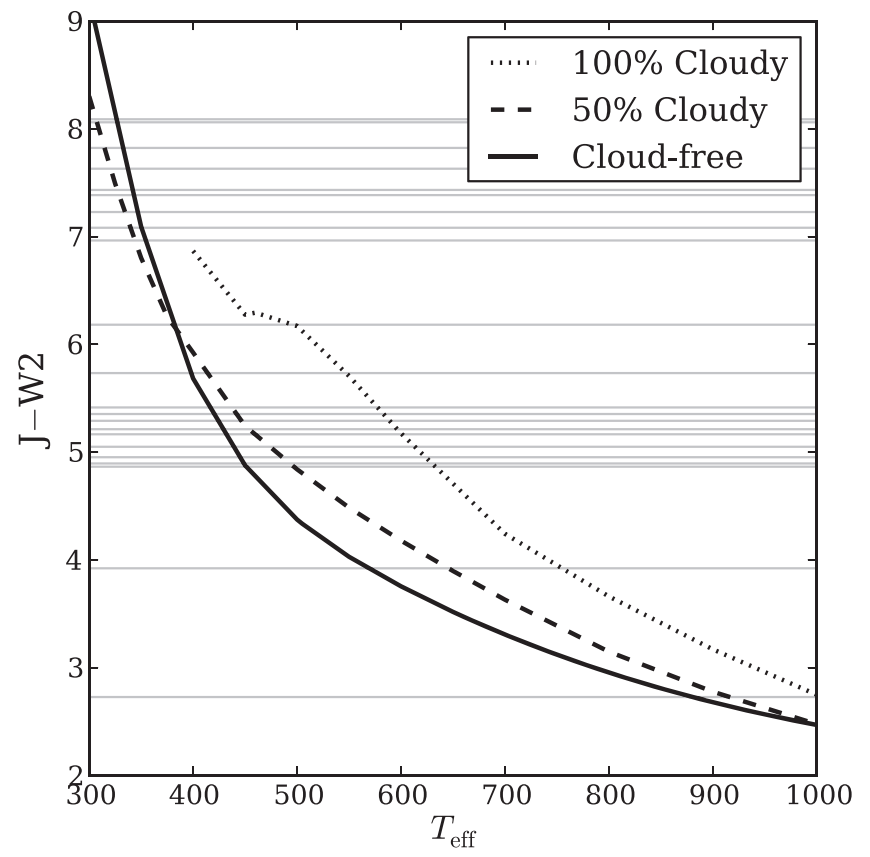

Figure 18. $J-W 2$ color as a function of effective temperature for a cloud-free model from Saumon et al. (2012) $(\log g=5)$, a $50 \%$ cloud coverage model from Morley et al. (2014) $\left(\log g=5, f_{\text {sed }}=5\right.$, with $\left.\mathrm{H}_{2} \mathrm{O}\right)$, and a $100 \%$ cloud coverage model from Morley et al. (2012) $\left(\log g=5, f_{\text {sed }}=3\right.$, without $\left.\mathrm{H}_{2} \mathrm{O}\right)$. Each horizontal gray line indicates a single $J-W 2$ value for a brown dwarf in our sample.

$g=5, f_{\text {sed }}=5$, with $\left.\mathrm{H}_{2} \mathrm{O}\right)$, and a $100 \%$ cloud coverage model from Morley et al. (2012) $\left(\log g=5, f_{\text {sed }}=3\right.$, without $\left.\mathrm{H}_{2} \mathrm{O}\right)$. Because our grid of models is, as of yet, incomplete (see Table 6), we show the only $50 \%$ cloud coverage model (with $\mathrm{H}_{2} \mathrm{O}$ ) for which the entire $300-1000 \mathrm{~K}$ temperature range is available, and the $100 \%$ cloud coverage model does not extend to temperatures $<400 \mathrm{~K}$. Even when limited to these three models, Figure 18 shows that effective temperature estimates for $J-W 2$ values less than $\sim 7$ typically have ranges greater than $100 \mathrm{~K}$. For $J-W 2$ values greater than $\sim 7$, varying percentages of cloud cover result in minimal differences in effective temperature, though the $100 \%$ cloudy model does not extend to this range. The range of temperatures for a single $J-W 2$ value will only increase with the inclusion of additional models with varying $\log g$ and $f_{\text {sed }}$ values.

Furthermore, as noted previously, the $J-$ band flux will also be affected by the reduced amount of $\mathrm{NH}_{3}$ present in the atmosphere due to vertical mixing (not only $Y$ and $H$ ). Figure 12 of Morley et al. (2014) displays equilibrium and disequilibrium models at 450, 300, and $200 \mathrm{~K}$. For the 450 $\mathrm{K}$ models, including disequilibrium chemistry will change the $Y_{-}, J_{-}$, and $H-$ band magnitudes by $\sim 0.2, \sim 0.3$, and $\sim 0.6$, respectively, implying that these near-infrared bands are affected by similar amounts when vertical mixing is included. Because of the large temperature ranges for individual $J-W 2$ values, uncertain temperature ranges for $J-W 2$ colors due to our incomplete model grid, and the similar influence of vertical mixing on the $J$ - band magnitude when compared to the $\mathrm{Y}$ and $\mathrm{H}$ bands, we conclude that estimating temperatures based solely on model $J-W 2$ colors is unsound. Since our observations are limited to near- and mid-infrared wavelengths and the current models do not include non-equilibrium chemistry, we lack a method to determine accurate effective temperatures for the objects in our sample.

Given the limitations of these particular models, determining effective temperatures by computing bolometric luminosities may therefore be preferable (e.g., Dupuy \& Kraus 2013). However, determining luminosities in this way currently relies on models to estimate the flux levels for up to $50 \%$ of the total luminosity (Dupuy \& Kraus 2013). Dupuy \& Kraus (2013) note that all current atmospheric models provide similar bolometric corrections in the wavelength ranges where flux has not yet been directly measured. Nevertheless, minimizing the dependence of the derived bolometric luminosities on the models should be a high priority, and therefore determining accurate effective temperatures for the lowest-mass brown dwarfs should focus on obtaining flux measurements at wavelengths longer than $\sim 5 \mu \mathrm{m}$. The near-infrared (NIRSpec and NIRCam) instruments and in particular the mid-infrared instrument (MIRI) aboard the forthcoming James Webb Space Telescope could prove invaluable in providing the additional flux coverage needed to determine accurate effective temperatures for the lowest-mass brown dwarfs.

\section{CONCLUSIONS}

We have presented $H S T$ near-infrared spectra for a sample of 22 brown dwarfs, six of which are new discoveries. Three of the new discoveries are classified as having spectral type Y, bringing the total number of spectroscopically confirmed $\mathrm{Y}$ dwarfs to 21. Theoretical spectra for the lowest-temperature brown dwarfs do not yet accurately reproduce the spectra from these observations. While disagreements between models and observations have been known to exist for these objects, the inclusion of HST G102 spectra has made discrepancies even more apparent. The primary disagreements occur in regions where models predict detectable $\mathrm{NH}_{3}$. These spectra, as well as those in Leggett et al. $(2013,2014,2015)$, show that the strong $\mathrm{NH}_{3}$ absorption features expected to occur in the $\mathrm{Y}$ - and $\mathrm{H}$ - 
band spectra of $\mathrm{Y}$ dwarfs are not present. While the nonequilibrium chemistry for $\mathrm{T}$ dwarfs reproduces observations quite well (Hubeny \& Burrows 2007), the very preliminary non-equilibrium models do not yet agree with the observations (Morley et al. 2014). That the models diverge from accurately describing the spectra of objects at such low temperatures begs for new models that include improved non-equilibrium chemistry, or the identification of another method for reducing $\mathrm{NH}_{3}$ abundances.

We wish to thank Caroline Morley and Didier Saumon for useful discussions regarding low-temperature models. This publication makes use of data products from the Wide-field Infrared Survey Explorer, which is a joint project of the University of California, Los Angeles, and the Jet Propulsion Laboratory/California Institute of Technology, and NEOWISE, which is a project of the Jet Propulsion Laboratory/California Institute of Technology. WISE and NEOWISE are funded by the National Aeronautics and Space Administration. This research has benefitted from the $\mathrm{M}, \mathrm{L}, \mathrm{T}$, and $\mathrm{Y}$ dwarf compendium housed at dwarfarchives.org. This research has benefitted from the SpeX Prism Spectral Libraries, maintained by Adam Burgasser at http://pono.ucsd.edu/ adam/ browndwarfs/spexprism. HST acknowledgement needed. We thank the STSCI help desk for useful discussions and resolution suggestions regarding WFC3 IR photometry. The authors wish to thank Caroline Morley for providing spectroscopic models via the webpage http://ucolick.org/ cmorley/ cmorley/Models.html.

\section{REFERENCES}

Beichman, C., Gelino, C. R., Kirkpatrick, J. D., et al. 2014, ApJ, 783, 68 Burgasser, A. J., Geballe, T. R., Leggett, S. K., Kirkpatrick, J. D., \& Golimowski, D. A. 2006, ApJ, 637, 1067

Burrows, A., Marley, M. S., \& Sharp, C. M. 2000, ApJ, 531, 438

Burrows, A., Sudarsky, D., \& Lunine, J. I. 2003, ApJ, 596, 587

Cushing, M. C., Roellig, T. L., Marley, M. S., et al. 2006, ApJ, 648, 614

Cushing, M. C., Marley, M. S., Saumon, D., et al. 2008, ApJ, 678, 1372

Cushing, M. C., Kirkpatrick, J. D., Gelino, C. R., et al. 2011, ApJ, 743, 50

Cushing, M. C., Kirkpatrick, J. D., Gelino, C. R., et al. 2014a, AJ, 147, 113
Dupuy, T. J., \& Liu, M. C. 2012, ApJS, 201, 19

Dupuy, T. J., \& Kraus, A. L. 2013, Science, 341, 1492

Fazio, G. G., Hora, J. L., Allen, L. E., et al. 2004, ApJS, 154, 10

Golimowski, D. A., Leggett, S. K., Marley, M. S., et al. 2004, AJ, 127, 3516

Gonzaga, S., Hack, W., Fruchter, A., \& Mack, J. (ed.) 2012, The DrizzlePac Handbook (Baltimore: STScI)

Hubeny, I., \& Burrows, A. 2007, ApJ, 669, 1248

Kimble, R. A., MacKenty, J. W., O’Connell, R. W., \& Townsend, J. A. 2008, Proc. SPIE, 7010, 43

Kirkpatrick, J. D. 2008, in ASP Conf. Ser. 384, 14th Cambridge Workshop on Cool Stars, Stellar Systems, and the Sun, ed. J. D. van Belle (San Francisco, CA: ASP) , 85

Kirkpatrick, J. D., Cushing, M. C., Gelino, C. R., et al. 2011, ApJS, 197, 19

Kirkpatrick, J. D., Gelino, C. R., Cushing, M. C., et al. 2012, ApJ, 753, 156

Kirkpatrick, J. D., Cushing, M. C., Gelino, C. R., et al. 2013, ApJ, 776, 128

Kirkpatrick, J. D., Schneider, A., Fajardo-Acosta, S., et al. 2014, ApJ, 783, 122

Kuntschner, H., Kümmel, M., Walsh, J. R., \& Bushouse, H. 2011, ST-ECF, Instrument Science Report WFC3-2011-05, 5

Leggett, S. K., Burningham, B., Saumon, D., et al. 2010, ApJ, 710, 1627

Leggett, S. K., Liu, M. C., Dupuy, T. J., et al. 2014a, ApJ, 780, 62

Leggett, S. K., Marley, M. S., Freedman, R., et al. 2007, ApJ, 667, 537

Leggett, S. K., Morley, C. V., Marley, M. S., et al. 2013, ApJ, 763, 130

Leggett, S. K., Morley, C. V., Marley, M. S., \& Saumon, D. 2015, ApJ, 799, 37

Liu, M. C., Delorme, P., Dupuy, T. J., et al. 2011, ApJ, 740, 108

Liu, M. C., Dupuy, T. J., Bowler, B. P., Leggett, S. K., \& Best, W. M. J. 2012, ApJ, 758, 57

Lodieu, N., Béjar, V. J. S., \& Rebolo, R. 2013, A\&A, 550, L2

Luhman, K. L. 2014, ApJ, 786, L18

Luhman, K. L., Burgasser, A. J., \& Bochanski, J. J. 2011, ApJ, 730, L9

Mace, G. N., Kirkpatrick, J. D., Cushing, M. C., et al. 2013, ApJS, 205, 6

Morley, C. V., Fortney, J. J., Marley, M. S., et al. 2012, ApJ, 756, 172

Morley, C. V., Marley, M. S., Fortney, J. J., et al. 2014, ApJ, 787, 78

Pinfield, D. J., Gromadzki, M., Leggett, S. K., et al. 2014, MNRAS, 444, 1931

Saumon, D., \& Marley, M. S. 2008, ApJ, 689, 1327

Saumon, D., Marley, M. S., Abel, M., Frommhold, L., \& Freedman, R. S. 2012, ApJ, 750, 74

Saumon, D., Marley, M. S., Lodders, K., \& Freedman, R. S. 2003, in IAU Symp. 211, Brown Dwarfs, ed. E. Martín (San Francisco, CA: ASP) , 345

Thompson, M. A., Kirkpatrick, J. D., Mace, G. N., et al. 2013, PASP, 125,809

Tinney, C. G., Faherty, J. K., Kirkpatrick, J. D., et al. 2012, ApJ, 759, 60

Tokunaga, A. T., \& Vacca, W. D. 2005, PASP, 117, 421

van Dokkum, P. G. 2001, PASP, 113, 1420

Vrba, F. J., Henden, A. A., Luginbuhl, C. B., et al. 2004, AJ, 127, 2948

Witte, S., Helling, C., Barman, T., Heidrich, N., \& Hauschildt, P. H. 2011, A\&A, 529, AA44 